\title{
High-order methods for the numerical solution of the BiGlobal linear stability eigenvalue problem in complex geometries
}

\author{
L. González ，V. Theofilis and S. J. Sherwin \\ School of Naval Engineering, Universidad Politecnica de Madrid Avd. Arco de la Victoria s/n, \\ 28040 Madrid, Spain \\ School of Aeronautics, Universidad Politecnica de Madrid Pza. Cardenal Cisneros 3, 28040 Madrid, Spain \\ Department of Aeronautics, Imperial College London, South Kensington Campus, London SW7 2AZ, U.K.
}

\begin{abstract}
SUMMARY
A high-order computational tool based on spectral and spectral/hp elements ( . Fluid. Mech. 2009; to appear) discretizations is employed for the analysis of BiGlobal fluid instability problems. Unlike other implementations of this type, which use a time-stepping-based formulation (J. Comput. Phys. $1994 ; 110(1): 82-102 ; J$. Fluid Mech. $1996 ; 322: 215-241$ ), a formulation is considered here in which the discretized matrix is constructed and stored prior to applying an iterative shift-and-invert Arnoldi algorithm for the solution of the generalized eigenvalue problem. In contrast to the time-stepping-based formulations, the matrix-based approach permits searching anywhere in the eigenspace using shifting. Hybrid and fully unstructured meshes are used in conjunction with the spatial discretization. This permits analysis of flow instability on arbitrarily complex 2-D geometries, homogeneous in the third spatial direction and allows both mesh $(h)$-refinement as well as polynomial $(p)$-refinement. A series of validation cases has been defined, using well-known stability results in confined geometries. In addition new results are presented for ducts of curvilinear cross-sections with rounded corners.
\end{abstract}

KEY WORDS: BiGlobal; stability; spectral elements; finite elements; complex geometries; eigenvalues

\section{INTRODUCTION}

The convergence properties of spectral methods, either using a Galerkin or pseudo-spectral projection [1,2], are put to optimal use in the context of global, also known as BiGlobal [3] or direct [4] instability analysis. Unlike low-order finite element methods, the order of convergence is not fixed and it is related with the maximum regularity of the solution. The reason for using spectral methods for this class of instability problems stems from the need to identify properties of the small-amplitude perturbations (e.g. frequency and amplification/damping rates) in an accurate manner. This is an increasingly difficult task when solving coupled systems of partial-differential equations as the Reynolds number increases and increasingly finer structures appear in the fluid.

In complex geometries, spectral/hp element methods [5] are of particular utility in analyzing instability, via both $h$ - and $p$-type refinements. When performing $h$-type refinement, a fixed-order

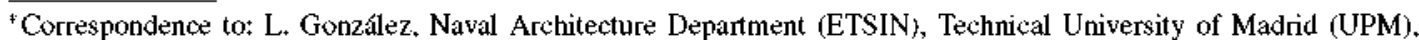
Arco de la victoria s/n, 28040 Madrid. Spain.

'E-mail: leo.gonzalez@upm.es
}

Contract/grant sponsor: Publishing Arts Research Council; contract/grant number: 98-1846389

Contract/grant sponsor: Universidad Politécnica de Madrid

Contract/grant sponsor: Colegio-Asociación de Ingenieros del ICAI 
polynomial is used in every element and convergence is achieved by reducing the size of the elements, $h$. On the other hand, $p$-refinement involves a fixed mesh and convergence is achieved by increasing the order of the polynomial in every element.

Highly accurate and geometrically flexible spectral $/ h p$ discretizations for the solution of linear instability problems in complex geometries, typically based on time-stepping methods, have an established history [6-8]. This approach has also been applied to a number of different complex geometry problems [9-13]. In this paper we present an alternative formulation undertaking a fluid mechanical instability analysis using the same class of discretizations, but constructing and storing the matrix system that arises from the discretized eigenvalue problem. The solution of the eigenvalue problem is then obtained by Krylov subspace iteration (Arnoldi) methods, after an LU-decomposition has been performed. Such an approach allows for the entire spectrum to be investigated using shifting techniques, although there is a (presently not quantified) computational cost implication when compared with the time-stepping formulation [7] for the leading eigenvalue. Exhaustive validation of the present formulation has been performed by investigating a series of well-known instability analysis applications in confined geometries. In addition new results are presented for ducts of curvilinear cross-sections with rounded corners.

The paper is organized as follows. In Section 2 the mathematical method is explained and some details of the numerical implementation are described. In Section 3, after presenting validation results on classic circular pipe and rectangular duct problems, the global instability of confined problems curvilinear intake geometries of technological interest in the motor-racing context is discussed. Concluding remarks are offered in Section 4.

\section{THEORY}

\subsection{Mathematical formulation}

The equations governing incompressible flows are written in primitive-variables $u_{i}, p$ formulation as follows:

$$
\begin{gathered}
\frac{\mathrm{D} u_{i}}{\mathrm{D} t}=-\frac{\partial p}{\partial x_{i}}+\frac{1}{\operatorname{Re}} \frac{\partial^{2} u_{i}}{\partial x_{j}^{2}} \text { in } \Omega, \\
\frac{\partial u_{i}}{\partial x_{i}}=0 \text { in } \Omega, \\
u_{i}=0 \text { on } \Gamma_{D} .
\end{gathered}
$$

Here $\Omega$ is the computational domain, $\Gamma_{D}$ is the boundary domain where in confined flows homogeneous Dirichlet boundary conditions are imposed, the material derivative operator is defined as usual,

$$
\frac{\mathrm{D}}{\mathrm{D} t}=\frac{\partial}{\partial t}+u_{j} \frac{\partial}{\partial x_{j}},
$$

and repeated indices imply summation. In a linear stability analysis context the steady laminar basic flow $\left(\bar{u}_{i}, \bar{p}\right)^{\mathrm{T}}$ is perturbed by small-amplitude velocity $\tilde{u}_{i}$ and kinematic pressure $\tilde{p}$ perturbations, as follows:

$$
u_{i}=\bar{u}_{i}+\varepsilon \bar{u}_{i}+c . c . \quad p=\bar{p}+\varepsilon \bar{p}+c . c .
$$

where $\varepsilon \ll 1$ and $c . c$. denotes conjugate of the complex quantities $\left(\tilde{u}_{i}, \tilde{p}\right)$ to force the real values of the velocity and pressure. Substituting into Equations (1)-(2), considering the basic 
flow as a particular solution of the Navier-Stokes equations, and linearizing, the equations for the perturbation quantities are obtained

$$
\begin{gathered}
\frac{\overline{\mathrm{D}} \tilde{u}_{i}}{\mathrm{D} t}+\tilde{u}_{j} \frac{\partial \bar{u}_{i}}{\partial x_{j}}=-\frac{\partial \tilde{p}}{\partial x_{i}}+\frac{1}{R e} \frac{\partial^{2} \tilde{u}_{i}}{\partial x_{j}^{2}}, \\
\frac{\partial \tilde{u}_{i}}{\partial x_{i}}=0,
\end{gathered}
$$

with

$$
\frac{\overline{\mathrm{D}}}{\mathrm{D} t}=\frac{\partial}{\partial t}+\bar{u}_{j} \frac{\partial}{\partial x_{j}}
$$

All the problems solved in this paper concern confined flows, for which the boundary condition used to close this system is

$$
\bar{u}_{i}=0 \quad \text { on } \Gamma_{D}
$$

In what follows, we seek numerical solutions to the system (5)-(7) for small-amplitude modal perturbations superimposed upon 1- and 2-D basic states. We monitor the former class of problems, for which abundant literature exists, in order to assess the accuracy of the numerical methods, before applying them to the BiGlobal problem.

2.1.1. 1-D EVP formulation and solution methodology. In the case of an 1-D basic state, with velocity components $(\bar{u}(y), 0,0)^{\mathrm{T}}$, the separability of temporal and spatial derivatives in $(5)-(6)$ permits introduction of an explicit harmonic temporal dependence of the disturbance quantities into these equations, according to the Ansatz

$$
\begin{gathered}
\tilde{u}_{j}=\hat{u}_{j}(y) \mathrm{e}^{\mathrm{i} \alpha x} \mathrm{e}^{\omega t}, \\
\tilde{p}=\hat{p}(y) \mathrm{e}^{\mathrm{i} x x} \mathrm{e}^{\omega t} .
\end{gathered}
$$

Here $i=\sqrt{-1}$ and $\alpha$ is a real wavenumber parameter, related with a periodicity length $L_{x}$ along the homogeneous direction through $L_{x}=2 \pi / \alpha$. For simplicity a 2-D perturbed flow is considered $\left(\hat{u}_{j}, \hat{p}\right)^{\mathrm{T}} \quad j=1.2$ are the complex amplitude functions of the linear perturbations. In a temporal context $\omega$ is the complex eigenvalue, the real and imaginary parts of which are, respectively, associated with the growth rate and the frequency of the perturbations. The case $\bar{w} \neq 0$ may be associated, using an appropriate transformation, with that in which $\bar{w}=0$, so that only the latter case is considered here. Substitution into (5)-(6) results in

$$
\begin{gathered}
\left\{\bar{u}_{j} \frac{\partial}{\partial x_{j}}-\frac{1}{R e}\left(\frac{\partial^{2}}{\partial x_{j}^{2}}-\alpha^{2}\right)\right\} \hat{u}_{1}+\hat{u}_{2} \frac{\partial \bar{u}_{1}}{\partial y}+\mathrm{i} \alpha p=-\omega \hat{u}_{1}, \\
\left\{\bar{u}_{j} \frac{\partial}{\partial x_{j}}-\frac{1}{R e}\left(\frac{\partial^{2}}{\partial x_{j}^{2}}-\alpha^{2}\right)\right\} \hat{u}_{2}+\frac{\partial \hat{p}}{\partial y}=-\omega \hat{u}_{2}, \\
\mathbf{i} \alpha \hat{u}_{1}+\frac{\partial \hat{u}_{2}}{\partial y}=0 .
\end{gathered}
$$

One defines

$$
a_{i i}=\left\{\bar{u}_{j} \frac{\partial}{\partial x_{j}}-\frac{1}{R e}\left(\frac{\partial^{2}}{\partial x_{j}^{2}}-\alpha^{2}\right)\right\}, \quad j=1,2 .
$$


using which the complex non-symmetric operator $A_{\text {an }}$ corresponding to the LHS of (10)-(12) may be defined as

$$
A_{\text {an } 1}=\left(\begin{array}{ccc}
a_{11} & \frac{\partial \bar{u}_{1}}{\partial y} & \mathrm{i} \alpha \\
0 & a_{22} & \frac{\partial}{\partial y} \\
\mathrm{i} \alpha & \frac{\partial}{\partial y} & 0
\end{array}\right) .
$$

After the Galerkin formulation. some details of which are presented in Section 2.2 and the Appendix A, the discrete operator becomes

$$
A_{1}=\left(\begin{array}{ccc}
R_{i j}+\alpha^{2} M_{i j}+\mathrm{i} \alpha E_{i j} & C_{i j} & \mathrm{i} \alpha D_{i j} \\
0 & R_{i j}+\alpha^{2} M_{i j}+\mathrm{i} \alpha E_{i j} & -\lambda_{i j}^{y} \\
\mathrm{i} \alpha D_{j i} & \lambda_{j i}^{y} & 0
\end{array}\right) .
$$

The real symmetric operator $B$ is also introduced by

$$
B_{1}=\left(\begin{array}{ccc}
M_{i j} & 0 & 0 \\
0 & M_{i j} & 0 \\
0 & 0 & 0
\end{array}\right),
$$

where $M$ represents the mass matrix; the elements of all matrices introduced in (15) and (16) are presented in the Appendix A. The system (10)-(12) is thus transformed into the (complex) generalized eigenvalue problem cEVP for the determination of $\omega$,

$$
A_{1}\left(\begin{array}{c}
\hat{u}_{1} \\
\hat{u}_{2} \\
\hat{p}
\end{array}\right)=-\omega B_{1}\left(\begin{array}{c}
\hat{u}_{1} \\
\hat{u}_{2} \\
\hat{p}
\end{array}\right) .
$$

The cEVP (17) has either real or complex solutions, corresponding to stationary $\left(\omega_{i}=0\right)$ or traveling $\left(\omega_{\mathrm{i}} \neq 0\right)$ modes. The real component $\omega_{\mathrm{r}}$ gives the growing or damping rate of the perturbation.

2.1.2. 2-D Laplace EVP formulation and solution methodology. The main target of the present paper is to investigate high-order Galerkin methods as applied to the solution of the BiGlobal EVP. However, prior to embarking upon this problem, the convergence properties of the proposed method are monitored on a simple 2-D EVP, the solution of which are analytically and/or numerically available [14]. The model problem considered is

$$
\begin{gathered}
-\nabla^{2} u+f(x, y) u=\frac{\partial u}{\partial t} \quad-1 \leqslant x, y \leqslant 1 \\
u=0 \quad \text { on } \Gamma_{D}
\end{gathered}
$$

The problem permits the introduction of an explicit harmonic temporal dependence of the disturbance quantities into this equation, according to the Ansatz

$$
\tilde{u}=\hat{u}(x, y) \mathrm{e}^{(0 t},
$$

In this case the operator $A_{\text {an }}$ is defined by

$$
A_{\text {an2 }}=\nabla^{2}-f(x, y) \text {, }
$$


After the Galerkin formulation, details of which are also presented in Section 2.2 and the Appendix A, the discrete operator $A$ becomes

$$
A_{2}=R_{i j}+F_{i j},
$$

The real symmetric operator $B$ is also introduced by

$$
B_{2}=M_{i j} \text {, }
$$

where $M$ represents the mass matrix; the elements of all matrices introduced in (22) and (23) are presented in the Appendix A. The system (18)-(19) is thus transformed into the (real) generalized eigenvalue problem for the determination of $\omega$,

$$
A_{2} \hat{u}=-\omega B_{2} \hat{u}
$$

The generalized eigenvalue problem (24) has either real solutions, corresponding to stationary $\left(\omega_{\mathrm{i}}=0\right)$ modes, or complex conjugate ones corresponding to traveling $\left(\omega_{\mathrm{i}} \neq 0\right)$ modes.

2.1.3. BiGlobal EVP formulation and solution methodology. Finally, attention is turned to the BiGlobal EVP. The basic flow velocity vector is now 2-D and may contain all three velocity components, $(\bar{u}, \bar{v}, \bar{w})^{\mathrm{T}}$, generalizing linear stability analysis to non-parallel flows [3]. For such basic states, the separability of temporal and spatial derivatives in (5)-(6) still permits introduction of an explicit harmonic temporal dependence of the disturbance quantities into these equations; however, the amplitude functions are now 2-D and the pertinent Ansatz is

$$
\begin{gathered}
\tilde{u}_{j}=\hat{u}_{j}\left(x_{1}, x_{2}\right) \mathrm{e}^{\mathrm{i} k x_{3}} \mathrm{e}^{(\omega t}, \\
\tilde{p}=\hat{p}\left(x_{1}, x_{2}\right) \mathrm{e}^{\mathrm{i} k x_{3}} \mathrm{e}^{\omega t t},
\end{gathered}
$$

where $i=\sqrt{-1}, k$ is a real wavenumber parameter, related with a periodicity length $L_{k}$ along the single homogeneous spatial direction through $L_{k}=2 \pi / k,\left(\hat{u}_{j}, \hat{p}\right)$ are the complex amplitude functions of the linear perturbations and $\omega$ is the complex eigenvalue that contains as the real part the growth/damping rate and as the imaginary part the frequency of the perturbation.

Substitution into (5)-(6) results in

$$
\begin{gathered}
\left\{\bar{u}_{j} \frac{\partial}{\partial x_{j}}-\frac{1}{R e}\left(\frac{\partial^{2}}{\partial x_{j}^{2}}-k^{2}\right)\right\} \hat{u}_{1}+\hat{u}_{2} \frac{\partial \bar{u}_{1}}{\partial x_{2}}+\hat{u}_{3} \frac{\partial \bar{u}_{1}}{\partial x_{3}}+\mathrm{i} k \hat{p}=-\omega \hat{u}_{1}, \\
\left\{\bar{u}_{j} \frac{\partial}{\partial x_{j}}-\frac{1}{R e}\left(\frac{\partial^{2}}{\partial x_{j}^{2}}-k^{2}\right)\right\} \hat{u}_{2}+\hat{u}_{3} \frac{\partial \bar{u}_{2}}{\partial x_{3}}+\frac{\partial \hat{p}}{\partial x_{2}}=-\omega \hat{u}_{2}, \\
\hat{u}_{2} \frac{\partial \bar{u}_{3}}{\partial x_{2}}+\left\{\bar{u}_{j} \frac{\partial}{\partial x_{j}}-\frac{1}{\operatorname{Re}}\left(\frac{\partial^{2}}{\partial x_{j}^{2}}-k^{2}\right)\right\} \hat{u}_{3}+\frac{\partial \hat{p}}{\partial x_{3}}=-\omega \hat{u}_{3}, \\
\mathrm{i} k \hat{u}_{1}+\frac{\partial \hat{u}_{3}}{\partial x_{3}}+\frac{\partial \hat{u}_{2}}{\partial x_{2}}=0 .
\end{gathered}
$$

In the presence of a basic flow velocity vector comprising components, $\left(\bar{u}_{1}, \bar{u}_{2}, \bar{u}_{3}\right)^{\mathrm{T}}$, one defines

$$
a_{i i}=\left\{\bar{u}_{j} \frac{\partial}{\partial x_{j}}+\frac{\partial \bar{u}_{i}}{\partial x_{i}}-\frac{1}{R e}\left(\frac{\partial^{2}}{\partial x_{j}^{2}}-k^{2}\right)+\mathrm{i} k \bar{u}_{1}\right\}, \quad j=1,2 .
$$


where no Einstein summation on the index $i$ is implied. The complex non-symmetric operator $A_{\text {an }}$ is defined by

$$
A_{\text {an } 3}=\left(\begin{array}{cccc}
a_{11} & \frac{\partial \bar{u}_{1}}{\partial x_{2}} & \frac{\partial \bar{u}_{1}}{\partial x_{3}} & \mathrm{i} k \\
0 & a_{22} & \frac{\partial \bar{u}_{2}}{\partial x_{3}} & \frac{\partial}{\partial x_{2}} \\
0 & \frac{\partial \bar{u}_{3}}{\partial x_{2}} & a_{33} & \frac{\partial}{\partial x_{3}} \\
i k & \frac{\partial}{\partial x_{2}} & \frac{\partial}{\partial x_{3}} & 0
\end{array}\right) .
$$

After the variational formulation. details of which are presented in appendix, the operator becomes

$$
A_{3}=\left(\begin{array}{cccc}
R_{i j}+\mathrm{i} k E_{i j} & C_{i j}^{12} & C_{i j}^{13} & \mathrm{i} k D_{i j} \\
0 & R_{i j}+C_{i j}^{22}+\mathrm{i} k E_{i j} & C_{i j}^{23} & -\lambda_{i j}^{y} \\
0 & C_{i j}^{32} & R_{i j}+C_{i j}^{33}+\mathrm{i} k E_{i j} & -\lambda_{i j}^{\overline{5}} \\
\mathrm{i} k D_{j i} & \lambda_{j i}^{y} & \lambda_{j i}^{j} & 0
\end{array}\right) .
$$

The real symmetric operator $B$ is also introduced by

$$
B_{3}=\left(\begin{array}{cccc}
M_{i j} & 0 & 0 & 0 \\
0 & M_{i j} & 0 & 0 \\
0 & 0 & M_{i j} & 0 \\
0 & 0 & 0 & 0
\end{array}\right) .
$$

where $M$ represents the mass matrix; the elements of all matrices introduced in (33) and (34) are presented in the appendix. The system (27)-(30) is thus transformed into the (complex) generalized eigenvalue problem for the determination of $\omega$,

$$
A_{3}\left(\begin{array}{c}
\hat{u}_{1} \\
\hat{u}_{2} \\
\hat{u}_{3} \\
\hat{p}
\end{array}\right)=-\omega B_{3}\left(\begin{array}{c}
\hat{u}_{1} \\
\hat{u}_{2} \\
\hat{u}_{3} \\
\hat{p}
\end{array}\right) .
$$

The complex generalized eigenvalue problem (35) has either real or complex solutions, corresponding to stationary $\left(\omega_{\mathrm{j}}=0\right)$ or traveling $\left(\omega_{\mathrm{j}} \neq 0\right)$ modes. In cases like the dict flow where the base flow has only one non-zero component in the homogeneous direction $x_{3}$, this is $\left(0,0, \bar{u}_{3}\left(x_{1}, x_{2}\right)\right)^{\mathrm{T}}$, the operator $A$ is unavoidably complex. On the other hand, if the base flow has two non-zero components $\left(\bar{u}\left(x_{1}, x_{2}\right), \bar{v}\left(x_{1}, x_{2}\right), 0\right)^{\mathrm{T}}$ in the plane orthogonal to the homogeneous direction $x_{3}$, the operator $A$ can be transformed into a real one, this transformation can be done, for example, in the lid-driven cavity (LDC) problem with the consequent reduction of memory requirements for the storage of the matrices $A_{3}$.

\subsection{Spectral/hp theory: modal discretization}

The numerical solution of the three EVPs described in the previous sections may be accomplished by a nodal expansion of the unknowns on a set of nodes, $x_{q}$, using a set of basis functions, $\Phi_{q}(x)$. Linear- and quadratic Lagrange polynomials are the method of choice for $\Phi(x)$ in low-order FEM 
[15], and have also been used in our earlier work [16]. The associated nodal points, $x_{q}$, are chosen such that $\Phi_{p}\left(x_{q}\right)=\delta_{p q}$, where $\delta_{p q}$ represents the Kronecker delta. This property implies that the discrete approximation, $u^{\delta}$, of a function may be defined at $x_{q}$ in terms of the expansion coefficients $\widehat{u}_{p}$ as

$$
u^{\delta}\left(x_{q}\right)=\sum_{p=0}^{P} \widehat{u}_{p} \Phi_{p}\left(x_{q}\right)=\sum_{p=0}^{P} \widehat{u}_{p} \delta_{p q}=\widehat{u}_{q} ;
$$

in other words, the expansion coefficients approximate the function at the set of the nodal points. However, experience with the low-order method has shown that the necessity to resolve structures associated with linear perturbations at moderate $R e$-numbers results in the need for rather fine grids, with all the consequent large memory and $\mathrm{CPU}$ time requirements [16].

It is then natural to seek an alternative high-order discretization, based on a modal expansion. The first characteristic of such an expansion is that there is no physical interpretation of the associated expansion coefficients. Second, modal expansion is hierarchical, meaning that the expansion set of order $P-1$ is contained within the expansion set of order $P$; a modal expansion based on the Legendre polynomials $L_{p}(x)$ will be used in what follows. The key property of this expansion set is its orthogonality which, in addition to the hierarchical construction, leads to well-conditioned matrices [5]. Note that, for problems involving up to second-order differentiation, as those encountered herein, it is sufficient to guarantee that the approximate solution is in $H^{1}$. Typically, in the finite element methods this is solved imposing a $C^{0}$ continuity between elemental regions, that is the global expansion modes are continuous everywhere in the solution domain while continuity in the derivatives is achieved at convergence [5]. Boundary and interior nodes are distinguished in this expansion: the former are equal to unity at one of the elemental boundaries and are zero at all other boundaries; the latter class of modes are non-zero only at the interior of each element and are zero along all boundaries. In the standard interval $\Omega=\{\xi \mid-1 \leqslant \xi \leqslant 1\}$ the $p$-type modal expansion is denoted by $\psi_{p}(x)$ and is defined as

$$
\psi_{p}(\xi)= \begin{cases}\frac{1-\xi}{2} & p=0 \\ {\left[\frac{1-\xi}{2}\right]\left[\frac{1+\xi}{2}\right] L_{p-1}(\xi)} & 0<p<P \\ \frac{1+\xi}{2} & p=P\end{cases}
$$

It may be seen that the lowest expansion modes, $\psi_{0}(x)$ and $\psi_{p}(x)$, are the same as the low-order finite element expansion, these boundary modes being the only modes that are nonzero at the ends of the interval. The remaining interior modes are zero at the ends of the interval and increase in polynomial order. As a consequence of the orthogonality of the Legendre polynomials $L_{p-1}(\xi)$ the stiffness and mass matrices are, respectively, tri- and penta-diagonal. The resulting discretization is denoted as spectral/hp element method [5].

In an 1-D spectral/hp decomposition the global expansion basis is decomposed into elemental subdomains that can then be mapped into the standard interval $[-1,1]$. The polynomial basis is then defined in the standard region. To complete a Galerkin formulation it will be necessary to choose some form of numerical integration; for the purpose of the present work Gaussian quadrature is selected. The 1-D concept may be extended to multiple dimensions in a straightforward manner. In two dimensions two standard regions, a quadrilateral or a triangle, may be used. All bases used in what follows can be expressed in terms of modified principal functions. In the quadrilateral expansion:

$$
\psi_{p q}\left(\xi_{1}, \breve{\zeta}_{2}\right)=\psi_{p}\left(\breve{\xi}_{1}\right) \psi_{q}\left(\xi_{2}\right)
$$

In the triangular expansion:

$$
\psi_{p q}\left(\xi_{1}, \xi_{2}\right)=\psi_{p}\left(\eta_{1}\right) \psi_{p q}\left(\eta_{2}\right)
$$


where $\psi_{p q}\left(\eta_{2}\right)$ is defined slightly different to the ones in [5, p. 111]. In our case to have a perfect matching with the Legendre polynomials these basis functions are defined as:

$$
\psi_{p q}(\eta)= \begin{cases}\psi_{q}(\eta) & p=0,0 \leqslant q \leqslant P \\ {\left[\frac{1-\eta}{2}\right]^{p+1}} & 1 \leqslant p \leqslant P, q=0 \\ {\left[\frac{1-\eta}{2}\right]^{p+1}\left[\frac{1+\eta}{2}\right] P_{q-1}^{2 p, 0}(\eta)} & 1 \leqslant p \leqslant P, 1 \leqslant q \leqslant P \\ \psi_{q}(\eta) & p=P, 0 \leqslant q \leqslant P\end{cases}
$$

In the standard quadrilateral region, the Cartesian coordinates $\left(\xi_{1}, \xi_{2}\right)$ are bounded by constant limits

$$
\mathscr{2}^{2}=\left\{\left(\xi_{1}, \xi_{2}\right) \mid-1 \leqslant \xi_{1}, \xi_{2} \leqslant 1\right\}
$$

This is not the case in the standard triangular region, where the bounds of the Cartesian coordinates $\left(\xi_{1}, \xi_{2}\right)$ depend on each other, that is,

$$
\mathscr{T}^{2}=\left\{\left(\xi_{1}, \xi_{2}\right) \mid-1 \leqslant \xi_{1}, \xi_{2}, \xi_{1}+\xi_{2} \leqslant 0\right\} .
$$

A means of developing a suitable tensorial-type basis within unstructured regions, such as the triangle, is suggested by Karniadakis and Sherwin [5]. in terms of a coordinate system in which the local coordinates have independent bounds. The advantage of such a system is that 1-D functions may be defined, upon which a multi-domain tensorial basis may be constructed. A suitable coordinate system. which describes the triangular region between constant independent limits, is defined by the transformation [5]

$$
\begin{gathered}
\eta_{1}=2 \frac{1+\xi_{1}}{1-\xi_{2}}-1, \\
\eta_{2}=\xi_{2} .
\end{gathered}
$$

The final step in constructing a multi-dimensional spectral/hp approximation is a mapping of every subdomain (element) into the corresponding standard region $[-1,1]$ for the 1-D case, $2^{2}$ for the 2-D quadrilateral elements or $\mathscr{T}^{2}$ for the 2-D triangular elements.

\subsection{Numerical aspects}

In order to ensure stability of the finite element dicretization of all EVPs solved, the inf-sup compatibility condition must be satisfied by the discrete spaces in which disturbance velocity components and disturbance pressure are, respectively, discretized. In the present spectral/hp solution the standard approach is not strictly followed [17], that is, the number of disturbance pressure modes has been kept one less than that of the disturbance velocities. The sparse structure of the operators has not been exploited, while all computations have been performed serially, on a $4.0 \mathrm{GHz}$ Intel P-IV PC. Details particular to each problem are discussed next.

2.3.1. 1-D EVP formulation and solution methodology. The relatively small size of this problem permits the use of the direct QZ algorithm, as implemented in the LAPACK [18] library, for the recovery of the complete spectrum. The calculation time is composed of two tasks, the construction of the matrices, $t_{m}$, and the solution of the generalized eigenvalue problem. $t_{e}$. The number of complex matrix elements of $A$ are

$$
(2(1+p n)+(1+n(p-1)))^{2}=(3-3 p n-n)^{2} \simeq(3 p n)^{2}=9 p^{2} N_{e}
$$


where $n=\sqrt{N_{e}}$. The approximate memory needed (in Mb) in order to allocate the main matrix $A$ with $N_{e}$ subdomains within $p$ order of approximation is

$$
\frac{8 \cdot 18}{1024^{2}} p^{2} N_{e}=144 N_{e}\left(\frac{p}{1024}\right)^{2}
$$

This permits increasing the order of the polynomial approximation beyond $p=500$, without encountering difficulties [19] in a typical modern personal computer. Nevertheless, it should be noted that the memory requirements increase quadratically with $p$ and linearly with $h$, meaning that it is advantageous to increase the number of subdomains, keeping a modest value of $p$, rather than increasing $p$ in order to achieve convergence. This is an essential difference of the present approach compared with the earlier works on the same problem $[19,20]$.

2.3.2. 2-D Laplace EVP formulation and solution methodology. Here too the QZ algorithm is used and the two main elements of CPU time consumption are again the time, $t_{m}$. necessary to form the operators $A$ and $B$ and the time, $t_{e}$, taken by the QZ algorithm. The operator $A$ in this case is composed of

$$
\left((1+p n)^{2}\right)^{2}=(1+p n)^{4} \simeq(p n)^{4}=p^{4} N_{e}^{2}
$$

real elements, where $n=\sqrt{N_{e}}$. The memory (in Mb) needed for the in-core storage of $A$ for $N_{e}$ subdomains at order $p$ is

$$
\frac{8 p^{4} N_{e}^{2}}{1024^{2}}=8 p^{4}\left(\frac{N_{e}}{1024}\right)^{2}
$$

Consequently, in this model 2-D EVP it becomes clear that the limitation of $4 \mathrm{~Gb}$ of available memory results in a $p \leqslant 150$.

2.3.3. BiGlobal EVP formulation and solution methodology. One of the particularities of the QZ algorithm in terms of memory requirements is its need to store both the discretized matrices $A$ and $B$, as well as two more matrices of equal size, if the computation of eigenvectors is required. Since no sparsity information is used by this algorithm, it becomes clear that the in-core storage becomes a limiting factor for the solution of the BiGlobal EVP [3]. In this respect, for the problem at hand the iterative Arnoldi algorithm has been implemented and the linear systems that need to be solved at each iteration have been solved by a direct dense LU decomposition [18]. A Krylov subspace dimension, $m=100$, has been kept fixed at all computations presented in what follows. In the light of this, the total CPU time required for the solution of the BiGlobal EVP is composed of three parts, the time, $t_{m}$. needed to calculate the operators $A$ and $B$; the time, $t_{L U}$. needed for the single LU decomposition of the $A$ matrix at the beginning of the iteration; and the time, $t_{A}$, needed for the Arnoldi algorithm. Regarding memory requirements (in Mb), the number of complex matrix elements needed to fill the dense operator $A$ is

$$
16 p^{4} N_{e}^{2} \text {. }
$$

The memory requirements are

$$
\frac{256 p^{4} N_{e}^{2}}{1024^{2}}=256 p^{4}\left(\frac{N_{e}}{1024}\right)^{2}=\left(\frac{16 p^{2} N_{e}}{1024}\right)^{2}
$$

Consequently, the maximum allocatable problem in a typical $4 \mathrm{~Gb}$ RAM memory machine is determined by the condition $N_{e} p^{2} \leqslant 4096$. It is worth noting that the computing cost in both CPU-time and memory requirements of the present spectral/hp methodology is similar to that of a spectral collocation solution of the complex EVP in the model problem solved [21], the latter numerical methodology being ideally suited for regular geometry at hand. The present method is expected to become substantially more competitive in stability analyses of flows in less regular domains. 


\section{RESULTS}

Several eigenvalue problems have been solved using the methodology discussed in the previous sections. The first is the classic linear instability of plane Poiseuille flow [22], which is addressed by the solution of the 1-D EVP in primitive-variables formulation. A model 2-D EVP with analytically known solutions [14] is solved next. The next two problems address BiGlobal linear instability in duct flows of rectangular and triangular cross-sections; the first of these is well-documented $[21,23]$ while the results in the latter problem are first obtained herein. A regularized cavity flow is analyzed next by a $\mathrm{Bi}$-Global linear instability considering a regularized boundary condition for the base flow [24]. Finally, two curvilinear cross section intakes have also been solved for the first time, demonstrating the ability of the method to deal with non-regular geometries [25].

\subsection{Modal linear instability in plane Poiseuille and Hagen-Poiseuille flows}

Solution of the generalized eigenvalue (17) problem includes results of the classic Orr-Sommerfeld equation (OSE) as well as stable modes corresponding to Squire's equation [26]. Results of the solution to the OSE, obtained by a low-order FEM method, are available [19] and serve for comparisons against those obtained by the present high-order primitive-variables spectral/hp element. Both results will be compared with the standard reference for this problem [22], which used spectral tau discretization and Chebyshev polynomials. For the plane Poiseuille flow the standard domain $y \in[-1,1]$ has been used, in which the 2-D basic flow, $\left(\bar{u}=1-y^{2}, \bar{v}=0\right)^{\mathrm{T}}$ is defined, on the other hand for the Hagen-Poisenille flow the standard domain $r \in[0,1]$ has been used, in which the 2-D basic flow, $\left(\bar{u}=1-r^{2}, \bar{v}=0\right)^{\mathrm{T}}$, is defined. Eigenvalue results at Orszag's critical parameters are presented in Table $I$. Convergence of $\omega_{\mathrm{r}}$ is achieved for $p \geqslant 60$ and, in a manner analogous to that of earlier related (but single-domain) work [19], no spurious eigenvalues have been encountered in the present combination of a Galerkin method and Legendre-polynomials basis functions. Figure 1 presents the critical eigenspectrum; making use of the possibility of the present method to increase the number of subdomains $h$, the polynomial degree approximation $p$ needed to obtain converged eigenvalue results could be reduced, as also can be seen in Table I. Figure 1 also present the dependence of the eigenspectrum on $p$ at the high Reynolds number value $R e=27000$ [19]. The bifurcation appearing at the lower part of the spectrum at $h / p=2 / 100$ is typical of lack of resolution for this (strongly damped) part of the S-family of eigenmodes [28] and disappears when increasing resolution by use of $h / p=2 / 150$. Interestingly higher quality results may be obtained by a single-domain computation, $h / p=1 / 200$, than by a two-domain computation using the same nominal resolution, $h / p=2 / 100$. Note also that results obtained point to algebraic convergence when increasing $h$ at constant $p$, and exponential convergence when increasing $p$ at constant $h$. Table II presents the dependence of the CPU time on $h$ and $p$; it may be seen that this time depends on a power $l \geqslant 1$ of $p$, while only linear dependence of the CPU time consumed on $h$ may be seen. From a point of view of efficiency, it is then preferable to attain convergence by increasing $h$ at some moderately high $p$, rather than keeping $h$ low (or unity) and use $p$ in order to converge the eigenvalues; while the CPU times involved in the solution of the present 1-D EVP are low, this rule-of-thumb will be useful in solving multi-dimensional

Table I. Most unstable eigenvalue obtained at critical conditions ( $R e=5772.22, \alpha=1.02056)$ of the plane Poiseuille flow PPF and at $(R e=1000, \alpha=1.0)$ of the Hagen-Poiseuille flow HPF using different combinations of $h$ and $p$. Reference results are $\omega=(5.9 E-10.0 .26400174)$ [22] for the PPF and $\omega=(-0.07086,0.84675)[27]$ for the HPF

\begin{tabular}{|c|c|c|c|c|c|c|c|c|c|c|c|}
\hline \multicolumn{8}{|c|}{ PPF } & \multicolumn{4}{|c|}{$\mathrm{HPF}$} \\
\hline$h$ & $p$ & $\omega_{\mathbf{r}}$ & $\omega_{\mathrm{j}}$ & $h$ & $p$ & $\omega_{\mathrm{r}}$ & $\omega_{\mathrm{i}}$ & $h$ & $p$ & $\left(t_{\mathrm{r}}\right.$ & $t_{\mathrm{j}}$ \\
\hline 1 & 30 & $1.35 E-5$ & 0.2640118409 & 2 & 30 & $2.87 \mathrm{E}-9$ & 0.2640017397 & 1 & 18 & -0.070764 & 0.846611 \\
\hline 1 & 40 & $-4.36 E-8$ & 0.2640017246 & 2 & 40 & $3.02 E-9$ & 0.2640017395 & 2 & 20 & -0.070802 & 0.846684 \\
\hline 1 & 60 & $3.02 \mathrm{E}-9$ & 0.2640017395 & 4 & 30 & $3.02 \mathrm{E}-9$ & 0.2640017395 & 2 & 22 & -0.070821 & 0.846705 \\
\hline 1 & 80 & $3.02 \mathrm{E}-9$ & 0.2640017395 & 4 & 40 & $3.02 \mathrm{E}-9$ & 0.2640017395 & 4 & 24 & -0.070858 & 0.846748 \\
\hline
\end{tabular}



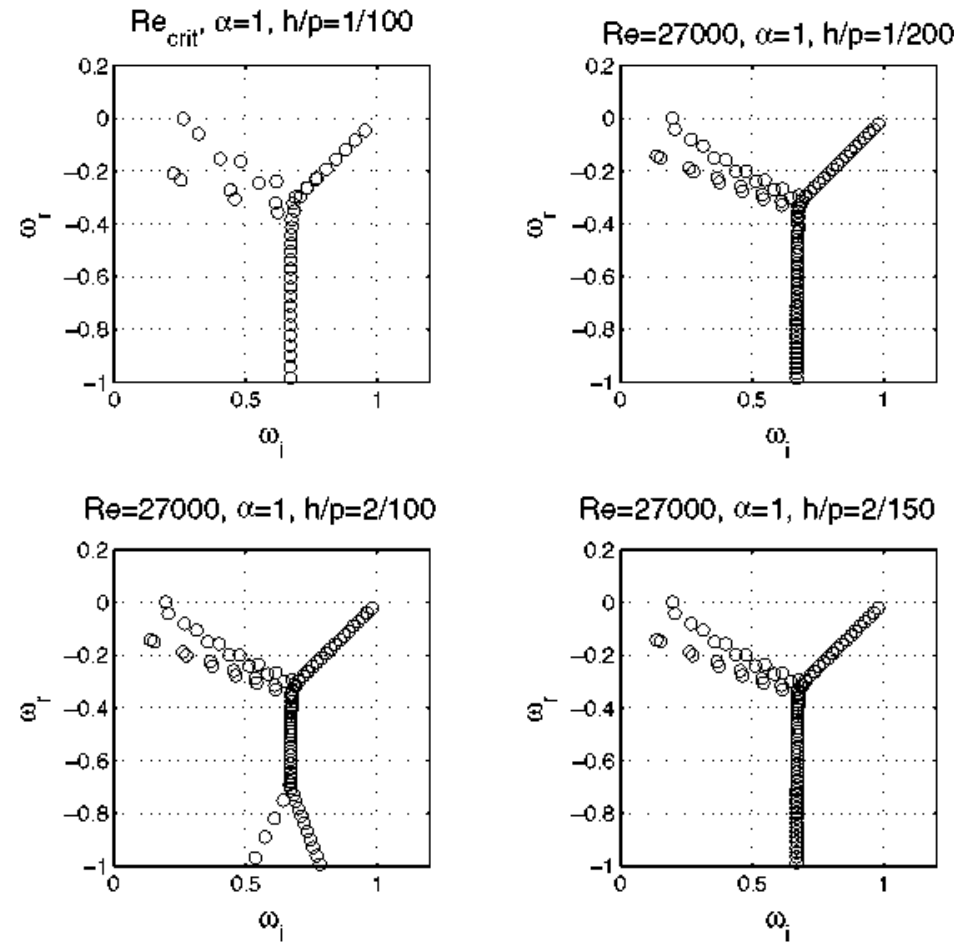

Figure 1. Spectra of the plane Poisenille flow at critical conditions $R e=R e_{c}$ and three different discretizations at $R e=27000, x=1$.

Table II. CPU-time at critical conditions of the plane Poiseuille flow, for different values of $h$ and $p$.

\begin{tabular}{rcrccccc}
\hline$h$ & $p$ & $t_{m}(\mathrm{~s})$ & $t_{e}(\mathrm{~s})$ & $h$ & $p$ & $t_{m}(\mathrm{~s})$ & $t_{\ell}(\mathrm{s})$ \\
\hline 1 & 20 & 0.05 & 0.02 & 2 & 30 & 0.52 & 0.42 \\
1 & 40 & 0.79 & 0.12 & 2 & 40 & 1.58 & 1.01 \\
1 & 60 & 3.78 & 0.41 & 4 & 30 & 0.97 & 3.64 \\
1 & 80 & 11.39 & 1.03 & 4 & 40 & 4.42 & 8.65 \\
\hline
\end{tabular}

EVPs. Changing from cartesian to polar coordinates, the same code has been used to calculate the axisymmetric eigenvalues of the Hagen-Poiseuille flow, see Table I. In this case the singularities at $r=0$ are avoided due to the use of Gauss points for the quadrature.

\subsection{2-D-Laplace eigenvalue problem}

The 2-D Laplace operator is at the heart of the linear operator describing small-amplitude BiGlobal instabilities. As such, the example solved in this section demonstrates the extensibility of the algorithms discussed in multiple dimensions and gain experience on a problem, the solution of which is known analytically. In particular, when $f=0,(18)$ and (19) may be solved by separation of variables, the eigenvalues being $\left(\pi^{2} / 4\right)\left(i^{2}+j^{2}\right) i, j=1,2,3 \ldots$ Table III demonstrates that a rather low value of the polynomial is capable of delivering the analytical result, while $h$-refinement plays a minor role in obtaining convergence. Still, order-of-magnitude reduction of the residuals is noticeable when doubling the number of domains, at the same constant $p=4$. The generalization of this case for bigger domains has been also calculated and the expected eigenvalues are obtained, these eigenvalues are equal to the $L=1$ case but divided into $L^{2}$. On the other hand, in the cases $f \neq 0$ the discrete matrix is less well-conditioned and substantially higher products $h \times p$ have to be used in order to converge the eigenvalues. Results not presented at low $h$ values demonstrate 
Table III. Convergence history of the Laplace EVP, for two different forcing functions and several combinations of $h$ and $p$.

\begin{tabular}{lcccccccc}
\hline$h$ & $p$ & $\omega_{\mathrm{T}}$ & $h$ & $p$ & $\omega_{\mathrm{T}}$ & $h$ & $p$ & $\omega_{\mathrm{T}}$ \\
\hline$f=0$ & & & & & & & & \\
1 & 2 & 2.0264236728 & 2 & 2 & 2.0150446547 & 4 & 2 & 2.0010242810 \\
1 & 4 & 2.0000294277 & 2 & 4 & 2.0000027311 & 4 & 4 & 2.0000000112 \\
1 & 6 & 2.0000000068 & 2 & 6 & 2.0000000000 & 4 & 6 & 2.0000000000 \\
1 & 8 & 2.0000000000 & 2 & 8 & 1.9999999999 & 4 & 8 & 2.0000000000 \\
$f=\exp (20(y-x-1))$ & & & & & & & \\
1 & 2 & 137.81737070 & 2 & 2 & 2.4515999319 & 4 & 2 & 2.1512062471 \\
1 & 4 & 2.2629988052 & 2 & 4 & 2.1484629835 & 4 & 4 & 2.1176838903 \\
1 & 6 & 2.1627748728 & 2 & 6 & 2.1247358185 & 4 & 6 & 2.1162728016 \\
1 & 8 & 2.1350717525 & 2 & 8 & 2.1180407896 & 4 & 8 & 2.1161928005 \\
1 & 10 & 2.1233966472 & 2 & 10 & 2.1167590461 & 4 & 10 & 2.1161849315 \\
\hline
\end{tabular}
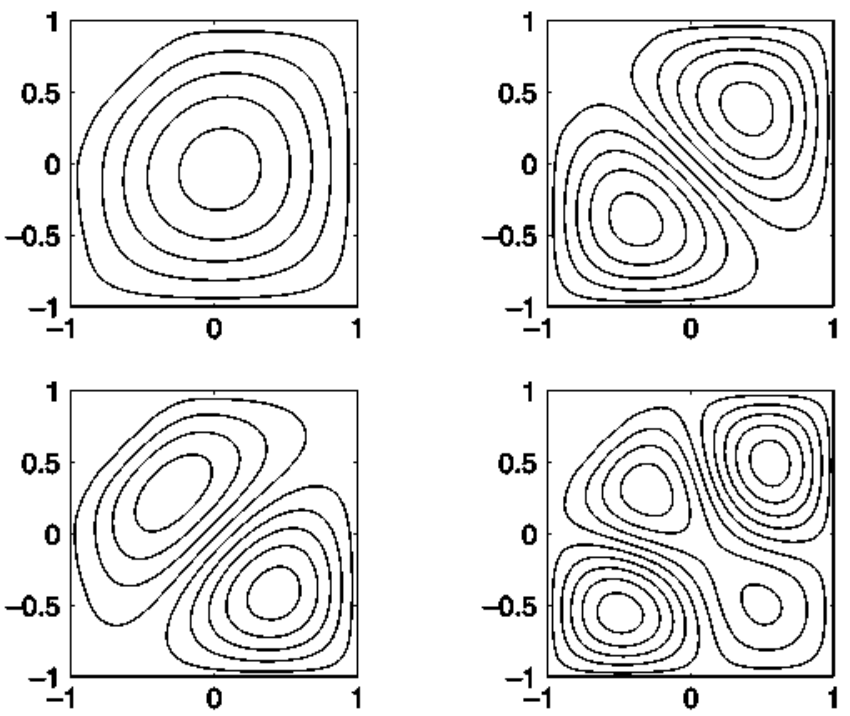

Figure 2. First four eigenvectors of (18)-(19) for $f(x, y)=\exp [20(y-x-1)][14]$

that, increasing $p$ at constant $h$ delivers convergence. However, (46) shows that increasing $p$ is less efficient in terms of memory requirements and associated CPU time demands than increasing $h$, the dependence of the memory on $p$ being quartic and that on $h$ being quadratic. As such, it is preferable to seek converged results by increasing $h$ at a moderate $p$. Contour plots of the first four eigenvectors of the $f \neq 0$ problem are shown in Figure 2; Table IV discusses that the computational times are measured versus $h$ and $p$.

\subsection{The BiGlobal instability analyses}

3.3.1. Hagen-Poiseuille flow. For this case and the following the variables will be renamed. $\left(x_{1}, x_{2}, x_{3}\right)=(y, z, x), k=\alpha,\left(\overline{u_{1}}, \overline{u_{2}}, \overline{u_{3}}\right)=(\bar{v}, \bar{w}, \bar{u})$. The algorithms exposed have been applied to study the stability of the classic Hagen Poiseuille flow (HPF). While, from a physical point of view, this is probably the most prominent example of failure of modal linear theory to predict transition, the corresponding 1-D eigenvalue problem (of the Orr-Sommerfeld class) has been studied exhaustively over the years $[27,29]$, thus serving for the present validation work. The analytically known basic flow, $\bar{u}=1-r^{2}$, has been recovered on the same unstructured mesh as that on which the BiGlobal instability analysis has been performed. Results on the eigenvalues of the two least-damped eigenmodes presented by Lessen [29] and Salwen [27] at four Reynolds numbers are shown in Table V, where excellent agreement with those results may be seen. The 
Table IV. CPU time for different combinations of $h$ and $p$.

\begin{tabular}{llll}
\hline$h$ & $p$ & $t_{m}(s)$ & $t_{\ell}(s)$ \\
\hline 1 & 4 & $1.2 \mathrm{E}-2$ & 0.000 \\
1 & 8 & 0.42 & $2.8 \mathrm{E}-2$ \\
2 & 4 & $3.2 \mathrm{E}-2$ & $2.8 \mathrm{E}-2$ \\
2 & 8 & 1.66 & 1.78 \\
4 & 4 & 0.11 & 1.92 \\
4 & 8 & 6.54 & 111.12 \\
\hline
\end{tabular}

Table V. Two least-damped eigennodes of Hagen-Poiseuille flow (HPF) at $k=\alpha=1$ for different Reynolds numbers, obtained on an single element mesh $h=1$ and $p$ polynomial degree.

\begin{tabular}{lccccc}
\hline$R e$ & Frequency & Damping rate & Frequency & Damping rate & \\
\hline 100 & 0.57256 & -0.14714 & 0.55198 & -0.37446 & Salwen [27] \\
100 & 0.57256 & -0.14714 & 0.55198 & -0.37446 & Lessen [29] \\
100 & 0.57256 & -0.14714 & 0.55198 & -0.37446 & Present $(h=1, p=18)$ \\
200 & 0.64427 & -0.12921 & 0.51116 & -0.20266 & Salwen [27] \\
200 & 0.64426 & -0.12920 & 0.51117 & -0.20265 & Lessen [29] \\
200 & 0.64526 & -0.12920 & 0.51117 & -0.20265 & Present $(h=1, p=20)$ \\
300 & 0.71295 & -0.12900 & 0.56173 & -0.16498 & Salwen [27] \\
300 & 0.71295 & -0.12907 & 0.56171 & -0.16497 & Lessen [29] \\
300 & 0.71295 & -0.12901 & 0.56172 & -0.16497 & Present $(h=1, p=22)$ \\
1000 & 0.84675 & -0.07086 & 0.46916 & -0.09117 & Salwen [27] \\
1000 & 0.84675 & -0.07086 & 0.46924 & -0.09090 & Lessen [29] \\
1000 & 0.84682 & -0.07090 & 0.46803 & -0.09033 & Present $(h=1, p=22)$ \\
\hline
\end{tabular}

corresponding amplitude functions are shown in Figure 3. The mesh utilized is superimposed in these figures, in which the following result of significance deserves further discussion. The mesh utilized has only one square element that has been mapped into a circumference [5], in which the following result of significance deserves further discussion. It must be remarked that without this curvilinear transformation it is extremely difficult to get an accurate approximation to the values found in the literature $[27,29]$, the only alternative could be approximating the circumference by straight lines but a very large number of elements should be used. This justifies the use of curvilinear transformations in those problems where complex geometries are analyzed. It must be remarked that using a single element in a circular pipe could be potentially problematic. When two curved edges in a quadrilateral element meet at a vertex with a continuous tangent the Jacobian of the mapping becomes singular. In our case the singularity is avoided by the use of quadrature rule that does not contain the boundary points like the Gauss' quadrature rule. This means that with this particular mapping the convergence rate could be affected.

In the context of classic linear stability theory, studied by numerical solution of the OrrSommerfeld equation, axial and azimuthal spatial homogeneity is assumed. The parameters of the problem are the Reynolds number, $R e$, and the axial and azimuthal wave numbers, $\alpha$ and $n$. At each Reynolds number, this theory obtains the amplitude functions of the eigenmodes as 1-D function of the radial coordinate and uses its Ansatz in order to reconstruct the spatial structure of the eigenmode. The eigenmodes of Figure 3 are identified as modes $n=1$ within the Orr-Sommerfeld theory, on account of the single-lobe structure of the amplitude functions. At each $R e$ and $\alpha$ within an Orr-Sommerfeld context, such results would have been obtained by the solution of an eigenvalue problem at $R e=100, \alpha=1 ; n=1$. In addition, the eigenvalue problems corresponding to $n=0$, $n \geqslant 2$ would need to be solved for a complete picture of the instability to be obtained at these $R e$ and $\alpha$ values. 

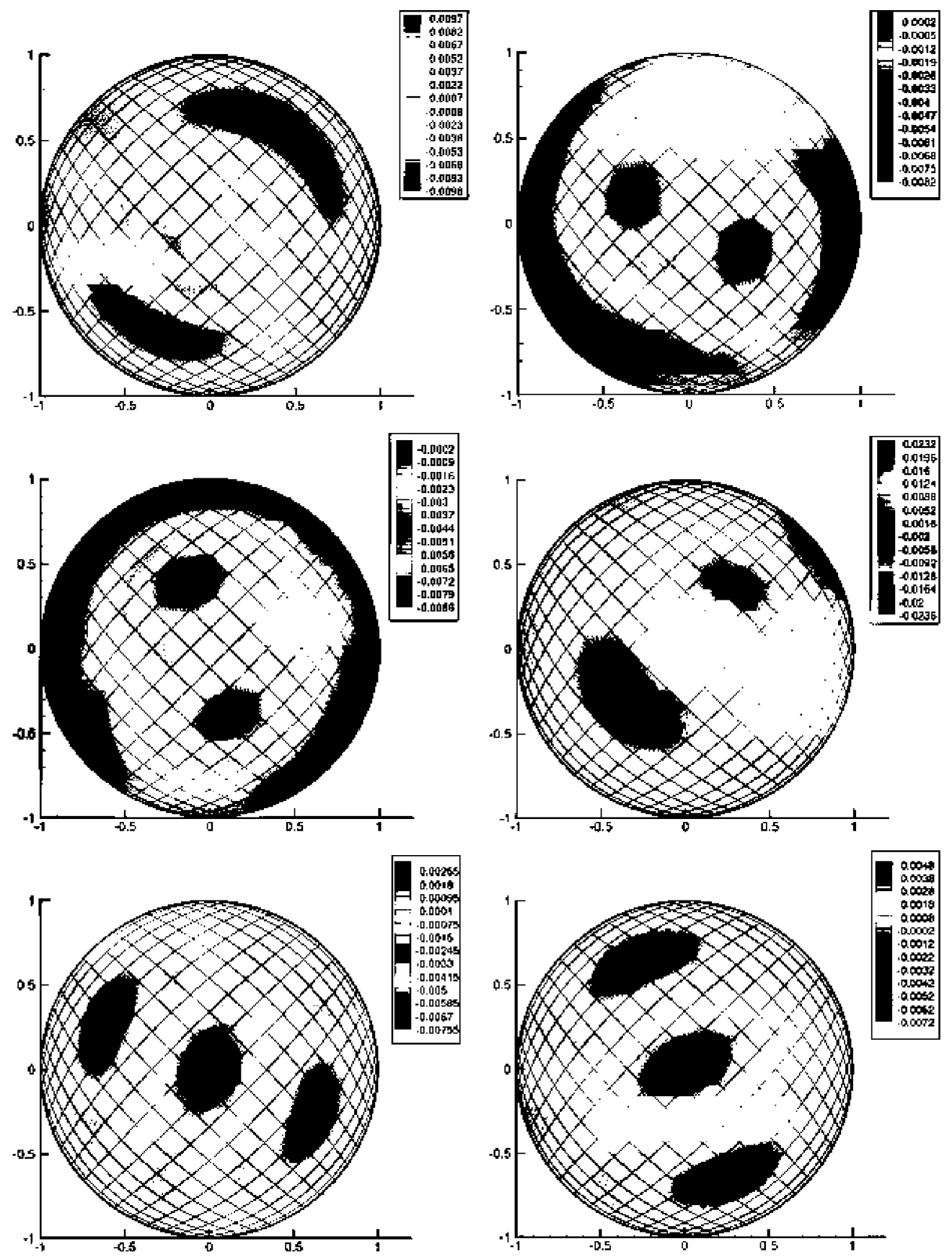

Figure 3. Amplitude functions of the perturbation velocity of the two least stable modes of HPF shown in Table $\mathrm{V}$ at $R e=100, \alpha=1$. Left to right column: $\Re\left\{\hat{u}_{1}\right\}, \Re\left\{\hat{u}_{2}\right\}$ (Upper row), $\Re\left\{\hat{u}_{3}\right\}, \Im\left\{\hat{u}_{1}\right\}$ (Middle row) and $\Im\left\{\hat{u}_{2}\right\}, \Im\left\{\hat{u}_{3}\right\}$ (Lower row). The mesh superposed shows the Gauss points used for the quadrature calculations in the single curved element $(h=1)$ that covers the entire domain.

By contrast, BiGlobal instability analysis only assumes axial homogeneity and resolves the entire plane normal to this direction. Its parameters are $R e$ and $\alpha$, i.e. are reduced by one compared with the classic theory. Within a BiGlobal context one solves one eigenvalue problem at each pair of $R e$ and $\alpha$ and the results known from classic Orr-Sommerfeld to correspond to distinct $n$ values may be identified in the BiGlobal eigenspectrum on account of the structure of the eigenfunction. In other words, it is stressed that both axisymmetric $(n=0)$ and multiple-lobed structures recovered in the eigenmodes of Figure 3 are the results of the BiGlobal analysis, not an assumption. 
3.3.2. Rectangular duct fow. Turning to the rectangular duct problem, the stability of which was first solved by Tatsumi and Yoshimura [23] is now going to be analyzed by the present method. Although spectral collocation would be an obvious option in order to discretize the spatial operator, in this case many other possibilities are available if spectral elements are used. A single element with increasing polynomial order has been used for the first convergence analysis, also other centered quadrilateral meshes and even hybrid meshes have been used for both the basic flow calculations and the instability analyses. The existence of a steady basic state implies stability of the 2-D $(\alpha=0)$ eigenmodes, such that the objective of the analysis becomes interrogation of the flow with respect to its stability to 3-D $(\alpha \neq 0)$ small-amplitude perturbations. Unlike the seminal work of Tatsumi and Yoshimura [23] on this problem the symmetries of the basic state have not been exploited in the instability analysis. The complex EVP to be solved here is (35), for which no reduction to a real problem is possible. The single component of the basic flow velocity vector, $(0,0, \bar{u}(y, z))$, is obtained from numerical solution (also using an spectral/hp element discretization) of the Poisson problem

$$
\begin{gathered}
\nabla^{2} \bar{u}(x, y)=-2, \\
\left.\bar{u}\right|_{\Gamma_{b}}=0 ;
\end{gathered}
$$

the maximum value of the basic state obtained has been used to normalize $\bar{u}$. Note that using the meshes necessary for convergence of the instability analysis results, the numerically obtained solution is identical with the (also numerically obtained) analytical solution of (49), see [30]

$$
\bar{u}(x, y)=\frac{1-y^{2}}{2}-\frac{16}{\pi^{3}} \sum_{k=1, k \text { odd }}^{\infty} \frac{\sin [k \pi(1+y) / 2]}{k^{3} \sinh k \pi}\left\{\sinh \left[\frac{k \pi(1+x)}{2}\right]+\sinh \left[\frac{k \pi(1-x)}{2}\right]\right\} .
$$

To confirm the spectral convergence of the method a single element mesh was studied for different polynomial order approximations at different Reynolds numbers. Results are presented in Table VI where the good convergence properties can be appreciated. In this case the base flow was calculated analytically by the expression (51). Error analysis of the damping rate and frequency results, using the values provided by a well-validated spectral collocation code [21] as a reference, show that convergence of the leading eigenvalue is obtained at a moderate polynomial order, $p=14$, and the relative error of the eigenvalue obtained compared with the spectral collocation result [21] of the same cEVP is of $O\left(10^{-6}\right)$. Results displayed in Figure 4 demonstrate exponential convergence.

A similar instability analysis have been repeated with an unstructured mesh using a baseflow coming from the solution of (49) in the square duct at $R e=100,1000,10000$, results are presented in Table VII where good accuracy has been obtained for the leading eigenmode increasing the $p$ value and keeping $h$ with a constant value. Finally, the computational solution in the general hybrid mesh described above has been also performed. The amplitude function of the leading damped eigenmode at $R e=100, \alpha=1$ can be seen in Figure 5.

In Table IX computational times are measured versus $h$ and $p$.

In this case the only geometrical degree of freedom that is present is the possibility of changing the aspect ratio $A R$, one of the critical points studied by [21,23] has also been calculated, that is $R e=10400, \alpha=0.91, A R=5$. The mesh used for the calculation is a $16(4 \times 4)$ element structured mesh with four elements per row and column. Calculations were done for two different polynomial approximations, obtaining for $p=18$ a imaginary part $\omega_{\mathrm{i}}=0.2126994 \mathrm{i}$ and for $p=20$ $\omega_{\mathrm{i}}=0.2115566 \mathrm{i}$, where good agreement can be appreciated $[21] \omega_{\mathrm{i}}=0.21167 \mathrm{i}$. The eigenvector corresponding to the critical mode is also shown in Figure 6.

The situation as far as the efficiency of the numerical approach is concerned changes as the Reynolds number increases. It is known that increasingly larger grids will be necessary in order to resolve the increasingly finer structures appearing as $R e$ increases.

3.3.3. Triangular duct fow. Two different triangular cross-section geometries have been analyzed. The first triangular duct $T 1$, which can be named as the canonical triangle in the spectral element 
Table VI. Least-damped eigenvalue in the square duct at $R e=100,1000, k=\alpha=1$, obtained at $h=1$ (single element) and different values of $p$. Reference values are [21, 23] $(R e=100, \omega=-0.140507+0.594177 \mathrm{i})$ and $(R e=1000, \omega=-0.065261+0.858880 \mathrm{i})$.

\begin{tabular}{cccccc}
\hline$h$ & $p$ & $\omega_{\mathrm{r}}$ & $\omega_{\mathrm{j}}$ & $\omega_{\mathrm{r}}$ & $\omega_{\mathrm{j}}$ \\
\hline 1 & 9 & -0.1416878659228 & 0.868727938313 & $-3.550714 \mathrm{E}-002$ & 0.36404258 \\
1 & 11 & -0.1403920660875 & 0.594072058091 & $-6.207683 \mathrm{E}-003$ & 0.47445694 \\
1 & 13 & -0.1404974881222 & 0.594182840228 & $-3.610017 \mathrm{E}-002$ & 0.56148818 \\
1 & 15 & -0.1404999231423 & 0.594175958418 & $-5.444386 \mathrm{E}-002$ & 0.47141951 \\
1 & 17 & -0.1404997246430 & 0.594175944273 & $-6.514139 \mathrm{E}-002$ & 0.85533862 \\
1 & 19 & -0.1404997260254 & 0.594175947962 & $-6.521440 \mathrm{E}-002$ & 0.85881800 \\
1 & 21 & -0.1404997258143 & 0.594175947500 & $-6.524627 \mathrm{E}-002$ & 0.85883308 \\
1 & 23 & -0.1404997256849 & 0.594175947325 & $-6.524283 \mathrm{E}-002$ & 0.85887825 \\
1 & 25 & -0.1404997256232 & 0.594175947240 & $-6.524303 \mathrm{E}-002$ & 0.85890439 \\
1 & 27 & -0.1404997255924 & 0.594175947194 & $-6.524305 \mathrm{E}-002$ & 0.85890387 \\
1 & 29 & -0.1404997255764 & 0.594175947170 & $-6.524308 \mathrm{E}-002$ & 0.85890028 \\
1 & 31 & -0.1404997255682 & 0.594175947156 & $-6.524310 \mathrm{E}-002$ & 0.85890062 \\
1 & 33 & -0.1404997255635 & 0.594175947148 & $-6.524316 \mathrm{E}-002$ & 0.85890098 \\
1 & 35 & -0.1404997255607 & 0.594175947144 & $-6.524319 \mathrm{E}-002$ & 0.85890010 \\
1 & 37 & -0.1404997255592 & 0.594175947141 & $-6.524321 \mathrm{E}-002$ & 0.85890115 \\
1 & 39 & -0.1404997255586 & 0.594175947139 & $-6.524322 \mathrm{E}-002$ & 0.85890125 \\
1 & 41 & -0.1404997255575 & 0.594175947138 & $-6.524322 \mathrm{E}-002$ & 0.85890122 \\
1 & 43 & -0.1404997255572 & 0.594175947137 & $-6.524322 \mathrm{E}-002$ & 0.85890123 \\
\hline
\end{tabular}

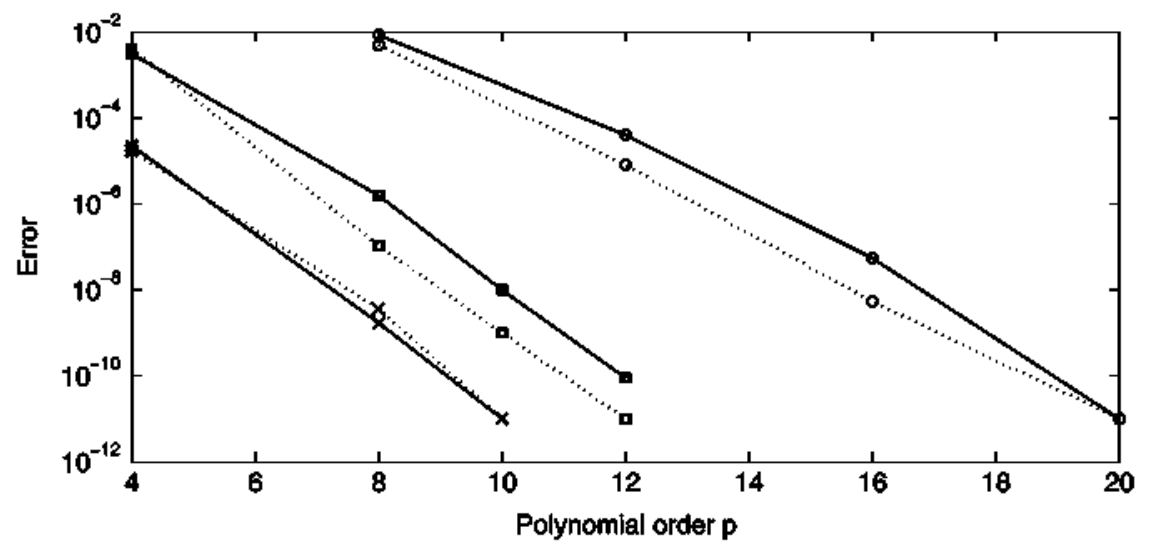

Figure 4. Error for the damping rate (continuous) and frequency (dotted) for the least damped eigenvalue at $R e=100, k=\alpha=1$, versus polynomial order for different meshes, [ $h=1$ (circles), $h=2^{2}$ (squares), $h=4^{2}$ (crosses)].

context, has its vertices at $(y=-1, z=-1),(y=-1, z=0)$, and $(y=0, z=-1)$. A second duct of equilateral triangular $T 2$ cross-section of unit side is defined, having its vertices at $(y=0, z=0)$, $(y=\sqrt{3} / 2, z=-0.5)$, and $(y=\sqrt{3} / 2, z=0.5)$. Flow driven by a constant pressure gradient along the homogeneous direction, $x$, through this duct has a single velocity component $\bar{u}(y, z)$; this velocity is obtained from the analytical solution of (49),

$$
\bar{u}(y, z)=-\frac{1}{\sqrt{3}}\left(y^{2}-3 z^{2}\right)\left(y-\frac{\sqrt{3}}{2}\right) .
$$

and normalized its midpoint value, $\bar{u}(y=\sqrt{3} / 3, z=0)=\frac{1}{18}$.

The existence of an exact solution of the Poisson equation for the non-trivial triangular geometry eliminates discretization errors in the instability analysis, originating from the description of the basic state, and thus facilitates monitoring the quality of the numerical tools proposed for the solution of the BiGlobal eigenvalue problem in this, somewhat academic, example. While for the 
Table VII. Least-damped eigenvalue in the square duct at $k=\alpha=1$ for different Reynolds numbers, in a unstructured mesh at different values of $p$. Reference values are $[21,23](R e=100, \omega=-0.140507+0.594177 \mathrm{i})$ and $(R e=1000, \omega=-0.065261+0.858880 \mathrm{i})$.

\begin{tabular}{|c|c|c|c|c|c|c|c|c|c|}
\hline \multirow[b]{2}{*}{$h$} & \multirow[b]{2}{*}{$p$} & \multicolumn{2}{|c|}{$R e=100$} & \multicolumn{2}{|c|}{$R e=1000$} & \multirow[b]{2}{*}{$h$} & \multirow[b]{2}{*}{$p$} & \multicolumn{2}{|c|}{$R e=10000$} \\
\hline & & $t_{\mathrm{r}}$ & $t_{j}$ & $t_{\mathrm{r}}$ & $t_{j}$ & & & $t_{\mathrm{r}}$ & $\omega_{\mathrm{i}}$ \\
\hline 5 & 5 & -0.13935459 & 0.5943767 & $-3.94148 E-002$ & 0.862675 & 16 & 14 & $-2.09064 \mathrm{E}-002$ & 0.955325 \\
\hline 5 & 7 & -0.14050700 & 0.5942498 & $-6.60404 \mathrm{E}-002$ & 0.865183 & 16 & 15 & $-2.09064 \mathrm{E}-002$ & 0.955322 \\
\hline 5 & 9 & -0.14050232 & 0.5941782 & $-6.57054 \mathrm{E}-002$ & 0.858584 & 16 & 16 & $-2.09063 \mathrm{E}-002$ & 0.955321 \\
\hline 5 & 11 & -0.14049988 & 0.5941767 & $-6.52441 \mathrm{E}-002$ & 0.858892 & 16 & 17 & $-2.09063 \mathrm{E}-002$ & 0.955319 \\
\hline 5 & 13 & -0.14049986 & 0.5941769 & $-6.52338 \mathrm{E}-002$ & 0.858911 & 16 & 18 & $-2.09063 \mathrm{E}-002$ & 0.955318 \\
\hline 5 & 15 & -0.14049986 & 0.5941769 & $-6.52235 E-002$ & 0.858913 & 16 & 19 & $-2.09063 \mathrm{E}-002$ & 0.955317 \\
\hline
\end{tabular}



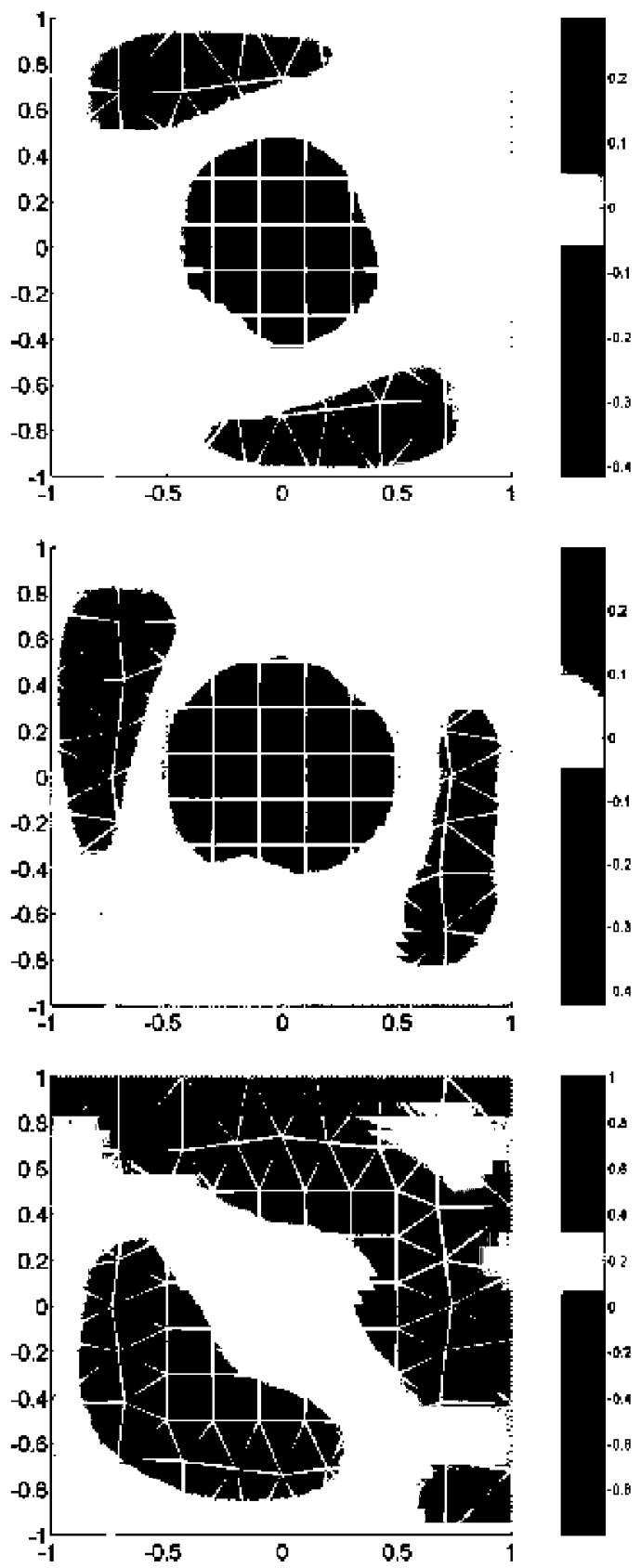

Figure 5. Components of the perturbation velocity of the leading (damped) mode at $R e=100, \alpha=1$ calculated on the superimposed hybrid mesh with $p=5$. Upper to lower: $\hat{u}, \hat{v}, \hat{w}$.

$T 1$ geometry the baseflow comes from the solution of the Poisson problem, in the second case $T 2$ the exact solution is used as the baseflow.

In Figure 8 the eigenvectors corresponding to the leading damped mode the $T 1$ case at $R e=100$ and $\alpha=1$. To validate this case, the same triangular duct was also analyzed with a second-order code with 23208 nodes and the same eigenvalues and eigenvectors were obtained.

Table VIII shows instability analysis results for the triangle $T 2$ at two different Reynolds numbers $R e=10^{2}, 10^{3}$, using a single triangular element, $h=1$, and an increasing polynomial degree, $p$, in order to obtain convergence. These results, completed with $R e=10^{4}$, are also represented in Figure 7 where the expected convergence properties can be appreciated. It must be remarked that 
Table VIII. Least-damped eigenvalue in the triangular duct $T 2$ at $R e=100, k=\alpha=1$ and $R e=1000, \alpha=1$, obtained at $h=\mathrm{I}$ and different values of $p$.

\begin{tabular}{llccc}
\hline$p$ & $\omega_{\mathrm{T}}$ & $\omega_{\mathrm{i}}$ & $\omega_{\mathrm{r}}$ & $\omega_{\mathrm{i}}$ \\
\hline 5 & -1.202437430325 & 0.3567409452173 & -0.95952131848 & 0.354820608 \\
7 & -1.193715846250 & 0.3742867553010 & -0.96044358510 & 0.399178849 \\
9 & -1.194274434585 & 0.3747834438871 & -0.96470987691 & 0.400266827 \\
11 & -1.19428156478 & 0.3747507106310 & -0.96488958403 & 0.4001055923 \\
13 & -1.19428101286 & 0.3747504491347 & -0.96488845751 & 0.4001002560 \\
15 & -1.19428099509 & 0.3747504266505 & -0.96488832692 & 0.4001006405 \\
17 & -1.19428099538 & 0.3747504271919 & -0.96488826155 & 0.4001006209 \\
19 & -1.19428099533 & 0.3747504272616 & -0.96488824129 & 0.4001006186 \\
21 & -1.19428099530 & 0.3747504272734 & -0.96488823516 & 0.4001006163 \\
23 & -1.19428099529 & 0.3747504272729 & -0.96488823340 & 0.4001006153 \\
25 & -1.19428099529 & 0.3747504272721 & -0.96488823288 & 0.4001006149 \\
27 & -1.19428099529 & 0.3747504272717 & -0.96488823273 & 0.4001006147 \\
29 & -1.19428099529 & 0.3747504272716 & -0.96488823268 & 0.4001006147 \\
\hline
\end{tabular}

Table IX. CPU times associated with solution of (33)-(34) at different combinations of $h$ and $p$.

\begin{tabular}{rrrrr}
\hline$h$ & $p$ & $t_{m}(\mathrm{~s})$ & $t_{L U}(\mathrm{~s})$ & $t_{A}(\mathrm{~s})$ \\
\hline 1 & 10 & 3.24 & 0.07 & 4.04 \\
1 & 20 & 272.64 & 2.72 & 21.75 \\
2 & 10 & 12.26 & 2.53 & 25.94 \\
4 & 10 & 52.33 & 128.77 & 135.69 \\
\hline
\end{tabular}

these triangles have less area Area $1=0.5$ and AreaT $2=1$ than any of the ducts analyzed and that is the reason because for the same $h$ and $p$ less error can be obtained, this way good convergence has been obtained even for relatively high Reynolds numbers, $\operatorname{Re}=10^{4}$.

3.3.4. Square regularized $L D C$. In this case the following variables will be renamed. $\left(x_{1}, x_{2}, x_{3}\right)=$ $(x, y, z), k=\beta,\left(\bar{u}_{1}, \bar{u}_{2}, \overline{u_{3}}\right)=(\bar{u}, \bar{v}, \bar{w})$ A square LDC domain $x \in[0,1] \times y \in[0,1]$ is defined. Flow is driven by the motion of the lid placed at $y=1$ along the positive $x$-direction, which results in a two-component basic velocity vector, $(\bar{u}(x, y), \bar{v}(x, y), 0)^{\mathrm{T}}$. The lid velocity is regularized according to $[16,24]$

$$
\bar{u}=\left(1-(2 x-1)^{18}\right)^{2} \text { at } y=1 \text {, }
$$

while $\bar{u}=0$ on the other three and $\bar{v}=0$ on all four cavity boundaries. Other regularization possibilities exist, notably the well-tested approximation of the singular LDC presented in [31], but have not been used here. It is worth noting that regularization of the boundary conditions in the cavity problem is essential in order to obtain a well-posed problem and avoid having to enter into the somewhat artificial debate found in the literature on the critical conditions for instability (cf. Poliashenko and Aidun [32] and related subsequent work) of a singular basic flow.

It must be also remarked that as we said at the end of Section 2.1 the complex operator $A$ could be transformed into a real one.

In order to ensure spatial convergence, a collocation Chebyshev spectral code has been used for the baseflow calculation, see details in [3]. The mesh considered for this particular case has [64 $\times 64]$ Gauss-Lobato collocation points.

With respect to the baseflow convergence in time, the relative tolerance

$$
\text { tol } \equiv \max _{i}\left\{\left|f_{i}(t+\Delta t)-f_{i}(t)\right|\right\}<10^{-15}
$$

has been used, where $f_{i}$ is the local value in a node $i$ of any flow quantity.

The instability problem in the regularized rectangular LDC has been solved by employing an EVP methodology based on numerical solution of (27)-(30) with a spectral/hp element discretization. 

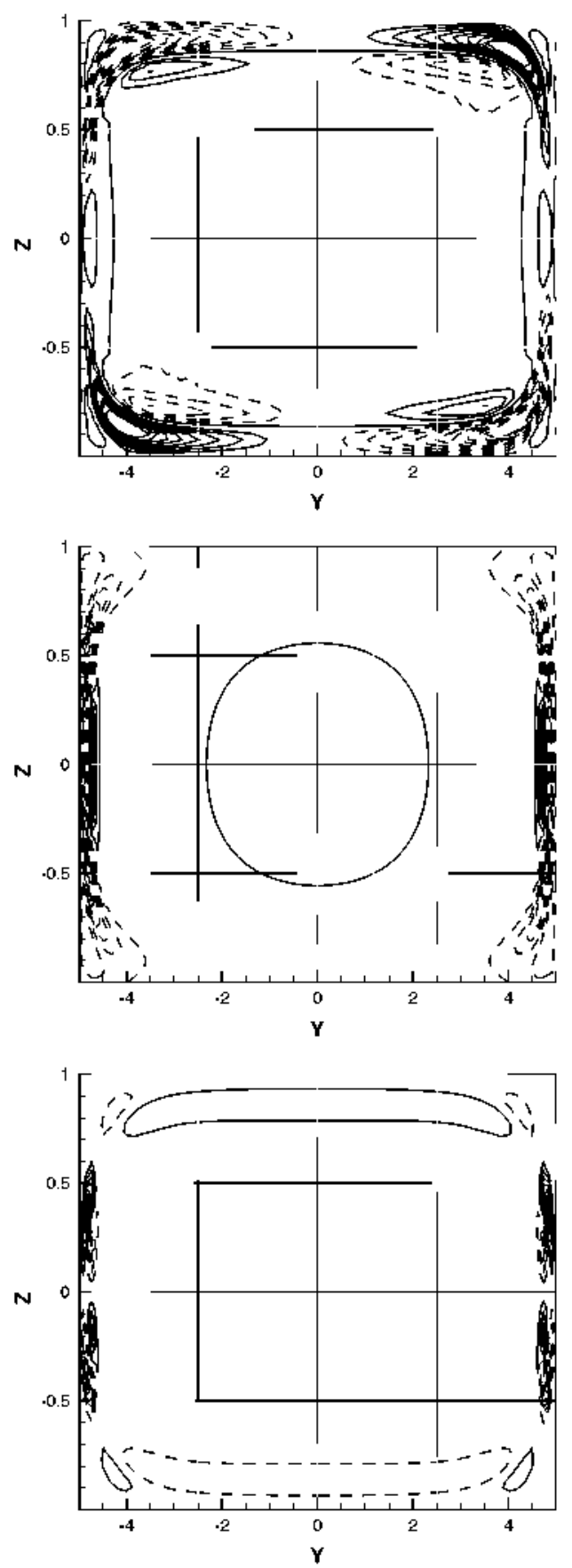

Figure 6. Components of the perturbation velocity of the critical mode at $R e=10400, \alpha=0.91$, $A R=5$ calculated on the superimposed structured mesh with $p=20$. Upper to lower: $\hat{u}: 19$ equispaced lines in interval $[-0.25,0.25], \hat{v}: 15$ equispaced lines in interval $[-0.23,0.17]$, $\hat{w}: 15$ equispaced lines in interval $[-1,1]$.

In addition, a spectral collocation algorithm [21,33] has been used for comparisons. Three Reynolds numbers were used in our simulations, two of them are stable $R e=200,800$, and the other unstable $R e=1000$. Different from the former problems presented, the grid has five elements $h=5$ distributed in a non-cartesian way. Four trapezoidal elements have been set around a central square element. This kind of centered meshes are often used in vortex stability calculations and it was a challenge to study their adequacy in cavity problems. Convergence results are presented 


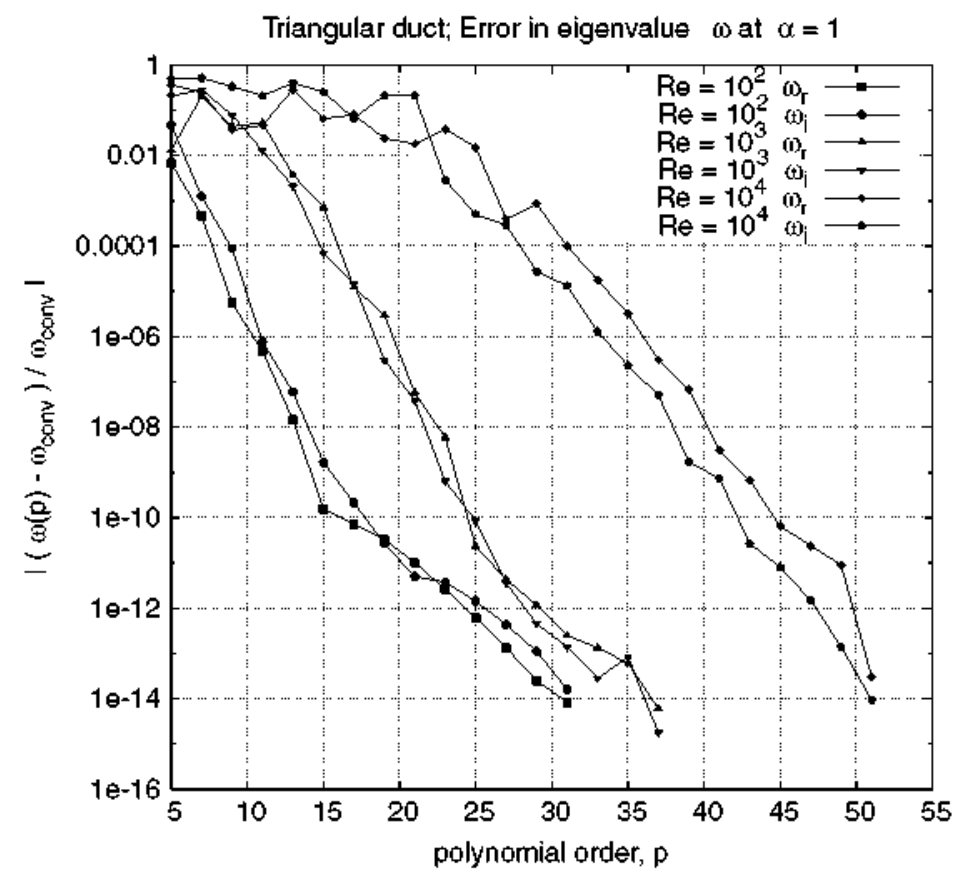

Figure 7 . Convergence of the real and imaginary parts of $\omega$ in the triangular duct $T 2$ at $\operatorname{Re}=100,1000,10000$ and $\alpha=1$.

for a certain region of the spectrum for $R e=1000$ and $R e=800$, see Figure 10 , in particular for the most unstable mode at $\beta=7.5$ good converge rate may be seen in Table $\mathrm{X}$ for both Reynolds numbers when the value of the polynomial order $p$ is increased.

Interestingly, at this Reynolds number the regularization condition (53) results in a general stabilization of the global eigenmodes, especially at large $\beta$ values, when compared with the standard LDC flow, in which the singular boundary condition $\bar{u}(x, y=1)=1$ is used in the place of (53). This result is in line with the analogous prediction of Theofilis [33], who analyzed a family of regularized profiles of the class discussed here. In addition, the frequency of the leading eigenmode is lower in the regularized compared with the singular LDC configuration; this effect also appears with the low-order finite element approximation [16]. The spatial distribution of the amplitude functions are qualitatively analogous; that of the leading eigenmode in the regulanized LDC flow at $(R e, \beta)=(1000,17)$ is shown in Figure 12 .

A consequence of the difference in amplification/damping rates among different cavity configurations is the increase of the linear critical Reynolds number pertinent to all known modes of the singular LDC, S1, T1, T2 and T3 [33]. In particular, with the regularization condition (53) the only two unstable modes were found at $R e=1000$ in the range $\beta \in[3,25]$ see Figure 11, while no unstable mode was found at $R e=1000$ with the boundary condition described in [16]. Decreasing the Reynolds number to 800 generate the stabilization of the two unstable modes that were found at $R e=1000$ with the (53) boundary condition. The dependence of the amplification rates of these modes, $\mathrm{T} 1$ and $\mathrm{S} 1$, on the spanwise wavenumber $\beta$ are shown in Figure 11 for different Reynolds numbers.

Finally, in Figure 9 the convergence rate of the least stable eigenvalue at $\operatorname{Re}=200, \beta=7.5$ is represented. It is remarkable that the good convergence properties are obtained in a problem where the baseflow and the analysis are performed with different codes and different meshes.

3.3.5. Curvilinear intakes. As all geometries presented up to this point are composed of a combination of straight lines or circumferences, it is desirable to close this analysis by performing a original complex geometry never analyzed before. 

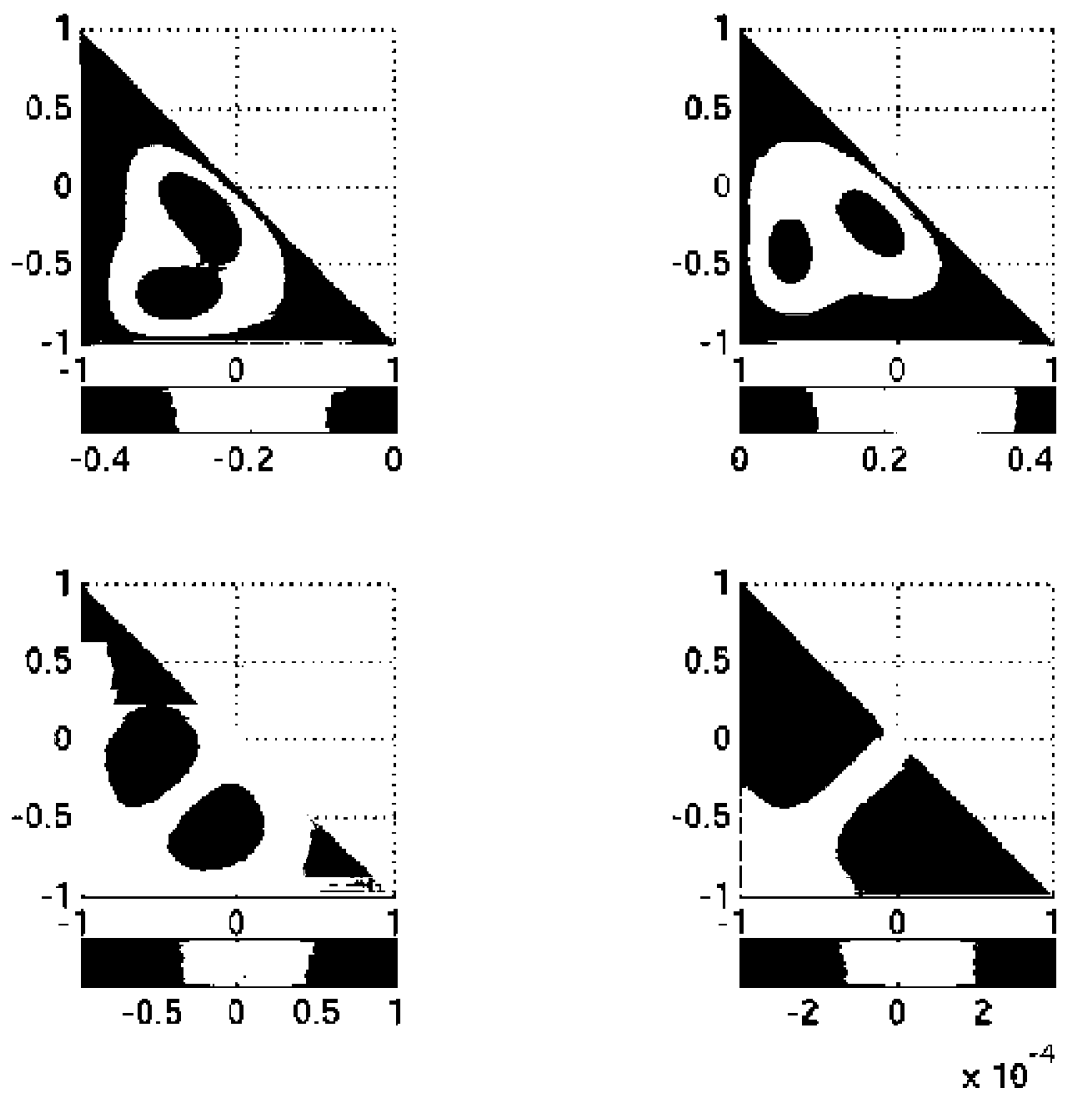

Figure 8 . Components of the perturbation velocity and pressure of the leading (damped) mode of the triangular duct flow $R e=100, k=\alpha=1.0$.

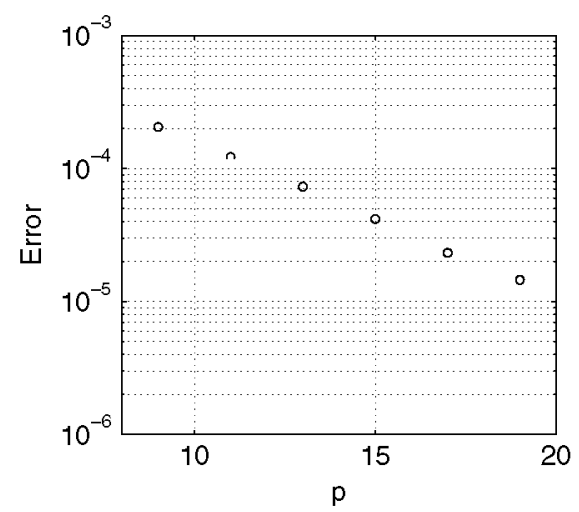

Figure 9. Error for the least stable eigenvalue in the cavity flow at $R e=200, \beta=7.5$.

The intake cross-sections are simplifications of real geometries, constructed using polygons and circumference arcs; all corners are replaced by circular arcs. The first intake in $_{1}$ is relevant for motor racing and has the shape of an isosceles triangle. The second intake $\mathbf{i n}_{2}$ is a model of those found on fighter aircraft and it is constructed as intersections of different-radii circles. The radii of the circular intake in ${ }_{2}$ are replaced by secant circular arcs. All corners are filleted with the same curvature radium $r$. The geometric parameters used, defined in Figure 13, are shown in Table XI. The meshes used for both intakes are shown in Figure 13 where it must be pointed out that no symmetry assumption has been taken into account. 


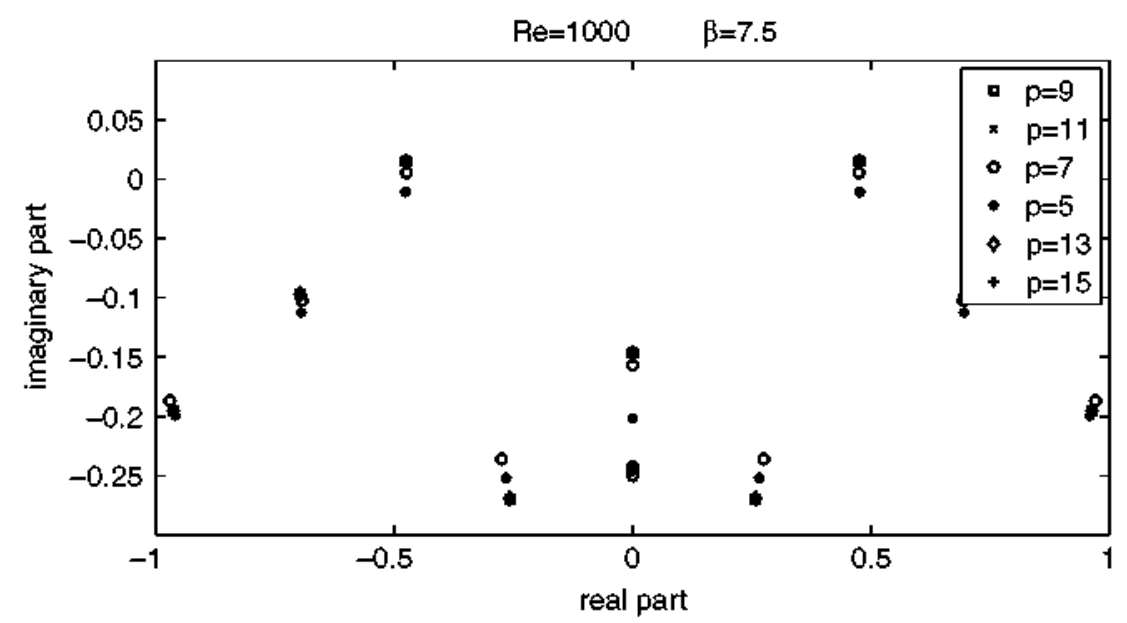

Figure 10. Most unstable part of the spectrum of the square cavity flow $R e=1000, k=\beta=7.5$.

Table X. Least-damped eigenvalue in the square cavity at $k=\beta=7.5$ for different Reynolds numbers, in a unstructured mesh at different values of $p$.

\begin{tabular}{ccccccc}
\hline & & \multicolumn{2}{c}{$R e=800$} & & \multicolumn{2}{c}{$R e=1000$} \\
\cline { 3 - 4 } \cline { 6 - 7 }$h$ & $p$ & $\omega_{\mathrm{T}}$ & $\omega_{\mathrm{i}}$ & & $\omega_{\mathrm{T}}$ & $\omega_{\mathrm{i}}$ \\
\hline 5 & 5 & $5.001 \mathrm{E}-002$ & 0.50807 & & $-1.090 \mathrm{E}-002$ & 0.47663 \\
5 & 7 & $3.203 \mathrm{E}-002$ & 0.50147 & & $-5.225 \mathrm{E}-002$ & 0.47511 \\
5 & 9 & $2.550 \mathrm{E}-002$ & 0.50270 & & $-1.482 \mathrm{E}-002$ & 0.47493 \\
5 & 11 & $2.543 \mathrm{E}-002$ & 0.50344 & & $-1.506 \mathrm{E}-002$ & 0.47592 \\
5 & 13 & $2.544 \mathrm{E}-002$ & 0.50340 & & $-1.507 \mathrm{E}-002$ & 0.47584 \\
5 & 15 & $2.544 \mathrm{E}-002$ & 0.50339 & & $-1.507 \mathrm{E}-002$ & 0.47583 \\
\hline
\end{tabular}

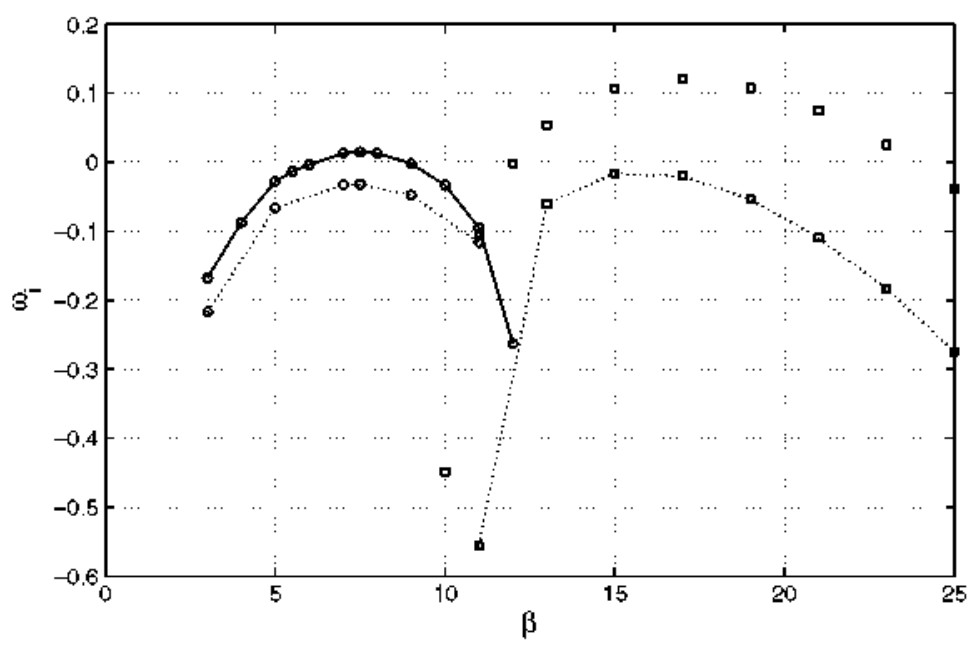

Figure 11. First two unstable modes $\mathrm{S} 1$ and $\mathrm{T} 1$ of the square cavity flow at $R e=1000$ (continuous) and $R e=800$ (dotted). S1(squares), T1(circles). 

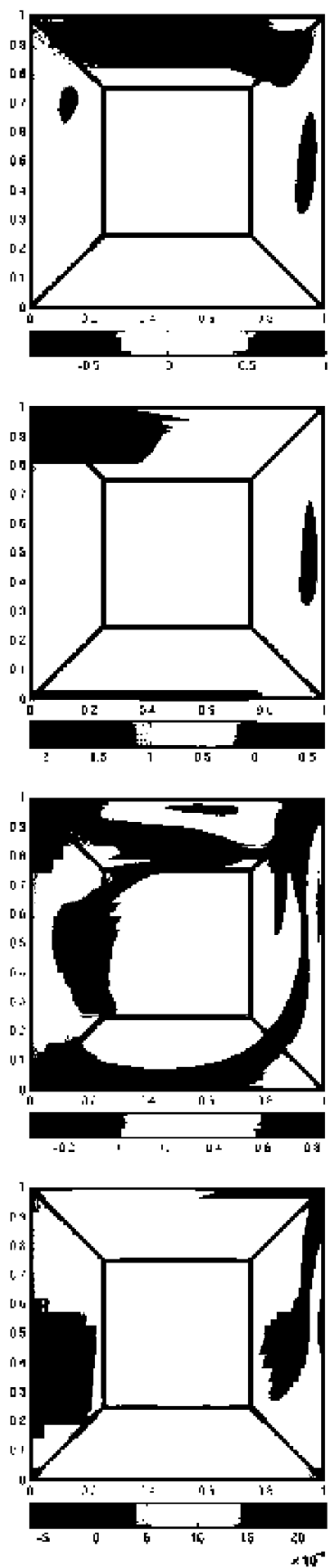

Figure 12. Most unstable mode of the square cavity flow at $R e=1000 \beta=17$.

From top to bottom: $\widehat{u}, \widehat{v}, \widehat{w}$ and $\widehat{p}$.

First, BiGlobal instability analyses have been performed for intake in 1 . Ten equispaced wavelengths from 1 to 10 have been analyzed at two different Reynolds numbers, $R e=100$ and $R e=1000$. Figure 14 presents the dependence of the damping rate and frequency on the streamwise wavenumber, indicating that $\alpha=3$ is the least stable wavelength. Figure 15 shows the amplitude functions of the disturbance velocity components pertaining to the leading (least damped) eigenmode at $R e=100$ and $\alpha=3$. The Reynolds number has then been increased an order of magnitude up to $R e=1000$ and a similar analysis has been performed. Figure 14 also presents the dependence of the damping rate and frequency on the streamwise wavenumber, indicating that when 

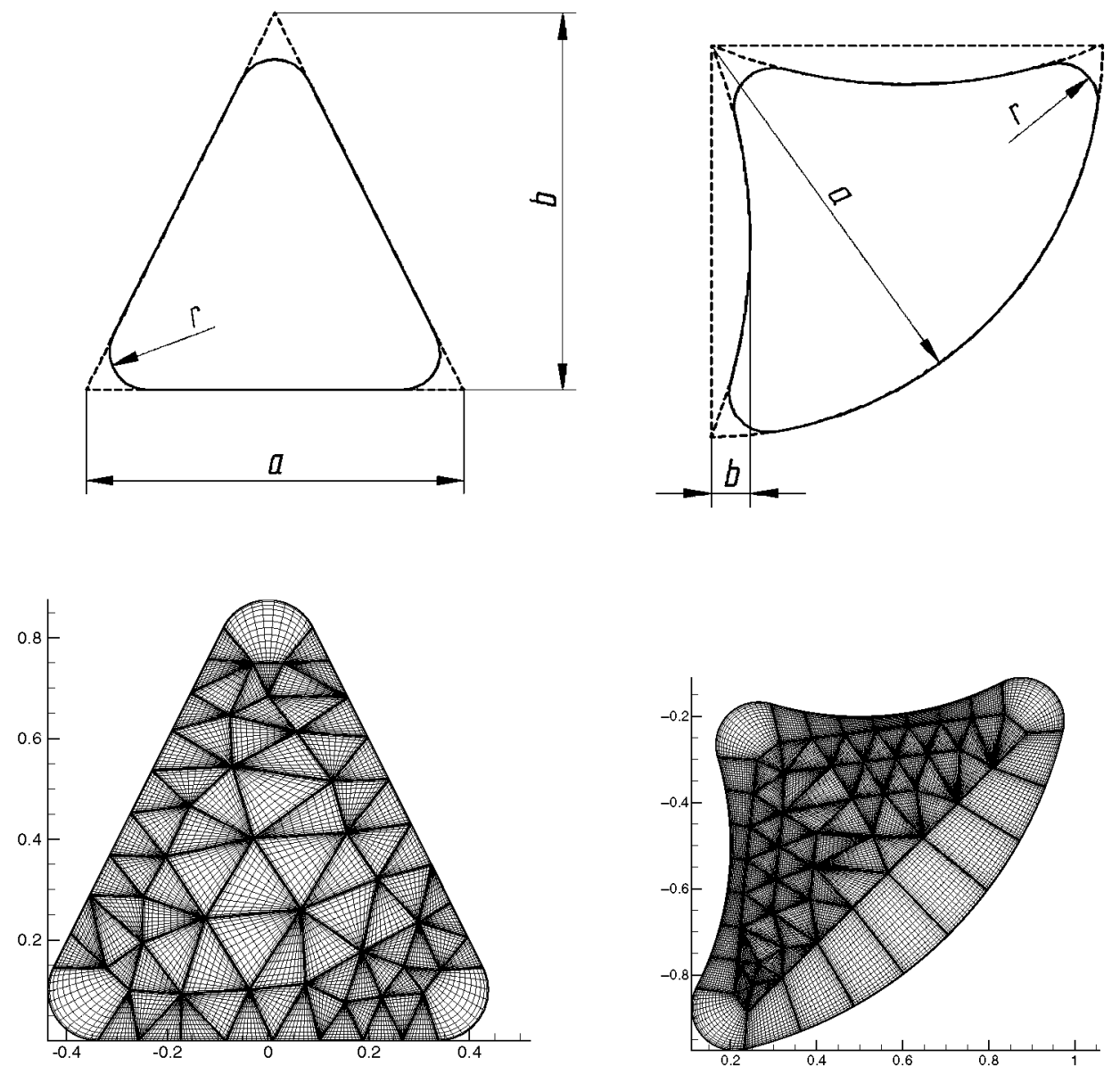

Figure 13. The two intake models analyzed, denoted as 'in ${ }_{1}$ ' and 'in '; the values of the respective parameters are shown in Table XI. Hybrid (triangles and quadrilaterals) meshes used for the two intakes models, denoted as 'in ' and 'in 2'; Gauss point grid (thin lines) and element grid (thick lines) [25].

Table XI. Geometrical definition $(a, b, r)$ and flow parameters (peak velocity, volume flux and area) of the intake models.

\begin{tabular}{lllcccc}
\hline Intake & $a$ & $b$ & $\mathrm{r}$ & $\max \left\{\bar{u}_{1}\left(x_{2}, x_{3}\right)\right\}$ & $Q$ & Area \\
\hline in $_{1}$ & 1 & 1 & 0.1 & 0.06371592 & 0.02970906 & 0.47929066 \\
in $_{2}$ & 1 & 0.2 & 0.1 & 0.06097295 & 0.02257703 & 0.47601238 \\
\hline
\end{tabular}

the wavelength decreases the leading eigenmode increases its damping rate, this tendency could mean that the 2-D flow is the least stable. While this is a potentially interesting result, in terms of the ability to analyze the complex flows at hand on the basis of relatively inexpensive 2-D (i.e. $\alpha=0$ ) BiGlobal analysis alone, many more results are necessary in order to confirm this finding. Comparing with the $R e=100$ case, it is possible to confirm that the absolute value of the damping rate decreases an order of magnitude something that could be qualitatively expected. Figure 15 shows the amplitude functions of the disturbance velocity components pertaining to the leading (least damped) eigenmode at $R e=1000$ and $\alpha=1$. The expected tightening of the structures associated with the increase of the Reynolds number may be appreciated in these results. While the first intake $i_{1}$ analyzed had a combination of straight lines and rounded corners, the second intake $\mathrm{in}_{2}$ has a completely curved perimeter. In order to perform an accurate calculation the analytical equation that describes the perimeter must be included as input information for the 

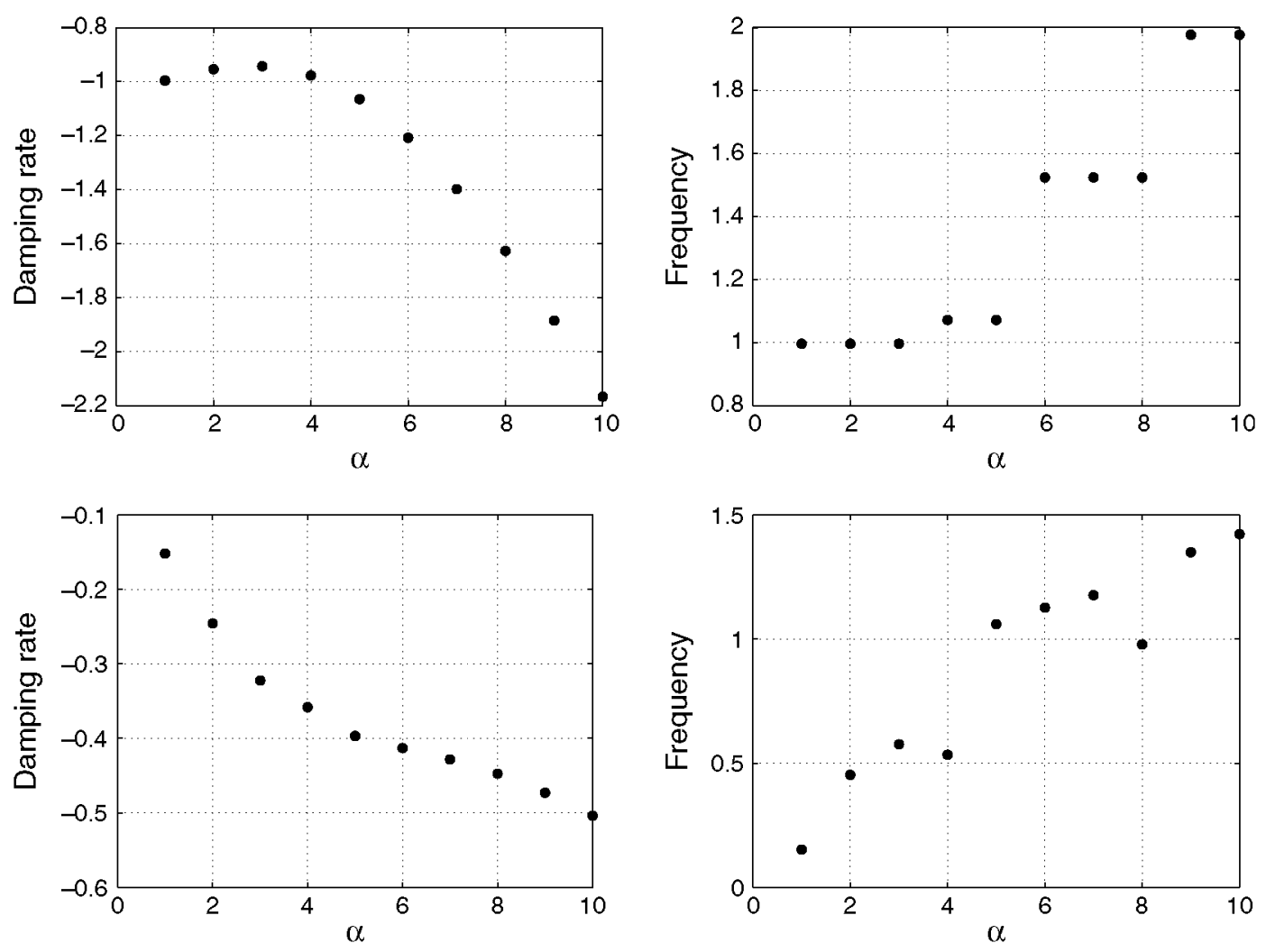

Figure 14. Dependence of damping rates (left) and frequency (right) on $\alpha$ at $R e=100$ (upper) and $R e=1000$ (lower) for 'in 1 '.
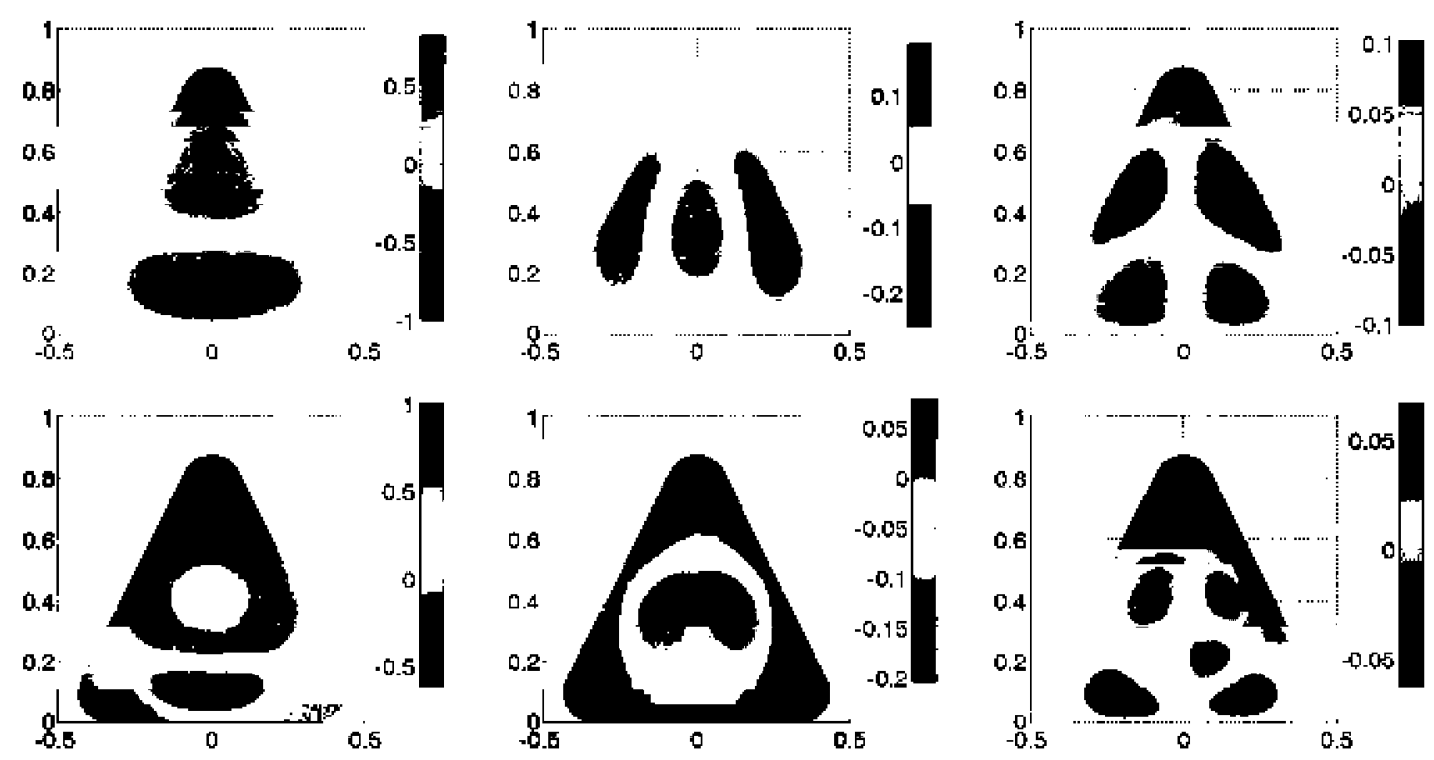

Figure 15. Amplitude functions of the least-damped eigenmode of 'in ' at $R e=100, \alpha=3$ (upper) and $R e=1000, \alpha=1$ (lower). Left to right column: $\hat{u}_{1}, \hat{u}_{2}, \hat{u}_{3}$.

code. Figure 16 presents the dependence of the damping rate and frequency on the streamwise wavenumber at $R e=100$, indicating that $\alpha=3$ is again the least stable wavelength. Comparing this result with the first intake we can see a slight decrease on the absolute value of the damping 

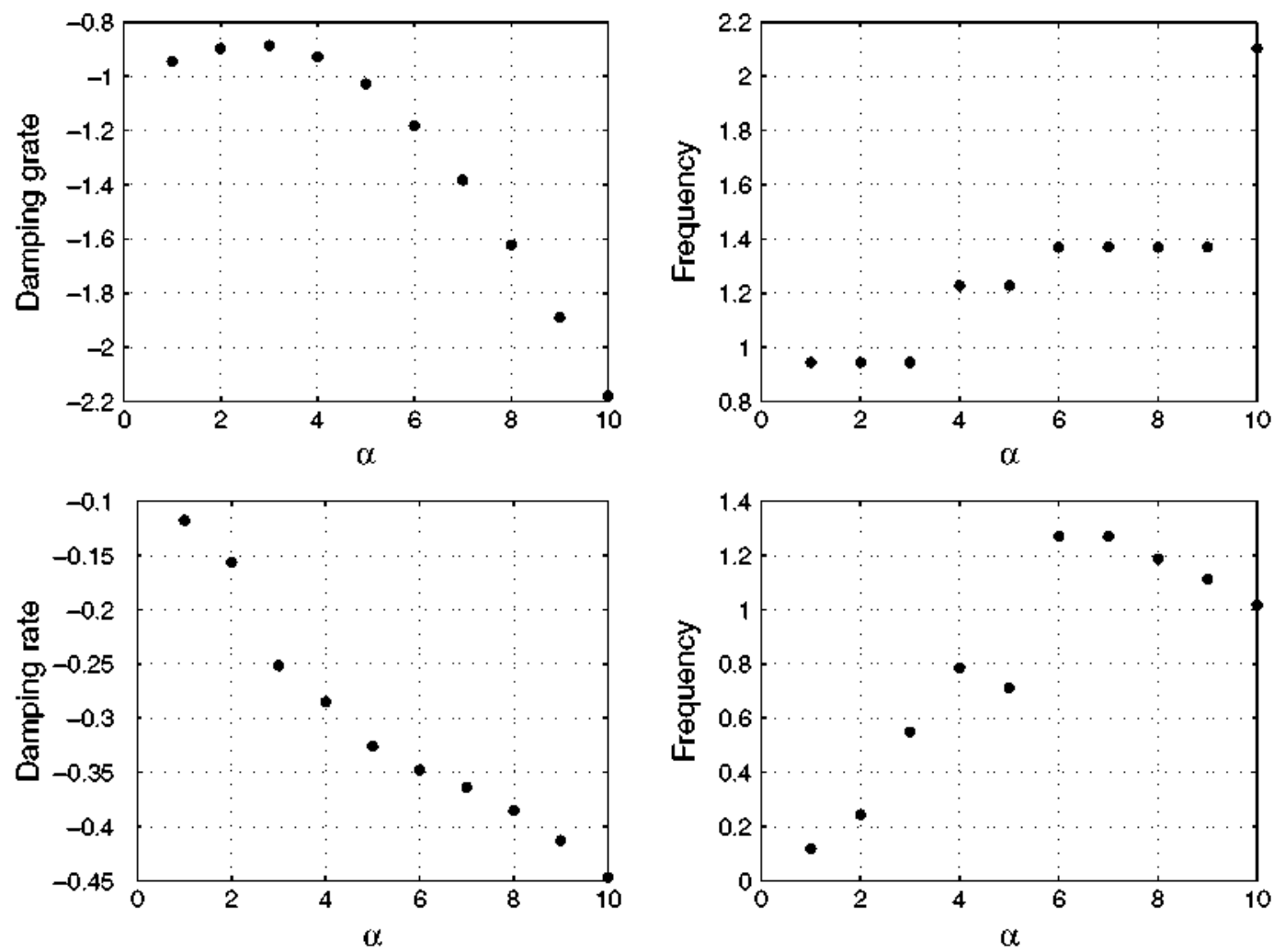

Figure 16. Dependence of damping rates (right) and frequency (left) on $\alpha$ at $R e=100$ (upper) and $R e=1000$ (lower) for 'in 2 '.

rate which means that the geometric change turned the intake $i_{2}$ into a less stable mode for the same Reynolds number. Figure 17 shows the amplitude functions of the disturbance velocity components pertaining to the leading (least damped) eigenmode at $R e=100$ and $\alpha=3$. Analogous results are produced when the Reynolds number is increased to $R e=1000$ for the second intake $i_{2}$, that is, less stable than the corresponding of both, the second intake in ${ }_{2}$ at $R e=100$ and the first intake $i_{1}$ at $R e=1000$. Figure 16 shows the dependence of the damping rate and frequency on the streamwise wavenumber. The mode corresponding to the least stable case can be found in 17. The symmetries in these results, existing on account of those of the corresponding basic state, are clearly visible. In line with the Hagen-Poiseuille flow analyzed earlier, these symmetries have not been imposed in the solution of the BiGlobal eigenvalue problem. However, exploitation of the symmetries is one obvious means of reducing the rather demanding memory requirements for the spectral/hp element method solution of the BiGlobal EVP as the Reynolds number increases.

\section{DISCUSSION}

In summary, the instability analysis examples presented establish the proposed methodology as a viable alternative to spectral collocation computations of BiGlobal instability in regular domains. The newly developed algorithms have a decisive advantage over the latter methodology when it comes to flexibility in handling geometric complexity. The proposed spectral/hp approach has been shown to combine accuracy and flexibility. Comparisons regarding the efficiency of the aforementioned established and the newly proposed methods, especially in terms of recovery of a wide window of the eigenspectrum, will be shown elsewhere. On the other hand, computational efficiency considerations aside, once sufficient resolution is provided, the method is capable of providing results in very good agreement with the established spectral computations. 

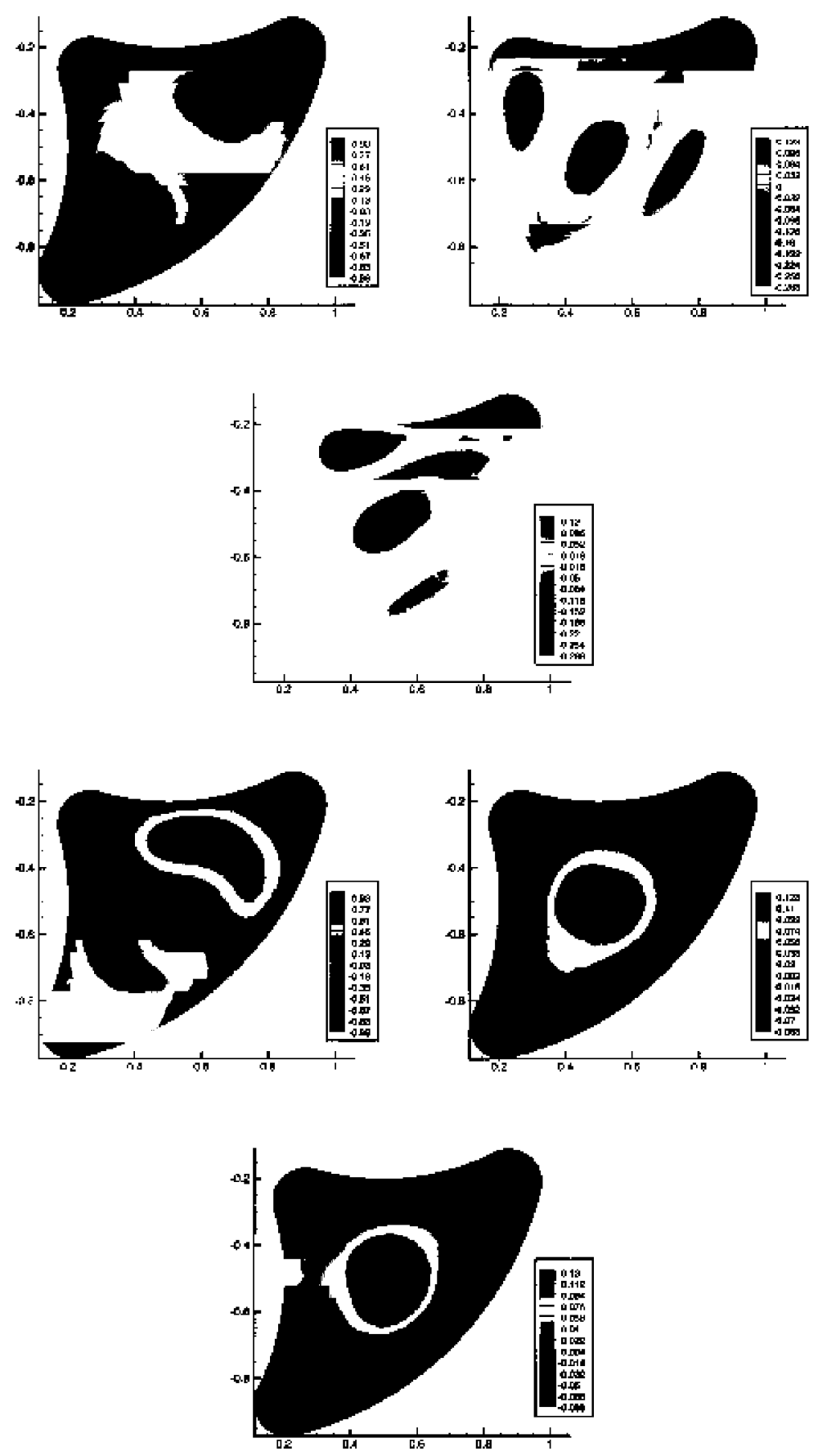

Figure 17. Amplitude functions of the least-damped eigenmode of 'in ' at $R e=100, \alpha=3$ (upper) and $R e=1000, \alpha=1$ (lower). Left to right column: $\hat{u}_{1}, \hat{u}_{2}, \hat{u}_{3}$.

Results in the previous sections have demonstrated the capacity of the proposed algorithms to address the problem that is at the heart of our investigations, linear modal instability of essentially non-parallel flows [3]. Three applications serve as demonstrators, the pressure-gradient-driven flow in a rectangular [23] and triangular duct and the instability of flow in a regularized-driven cavity of square cross-section $[16,24]$. Interesting, in the present context, is the fact that no spectral/hp element solutions of these BiGlobal EVPs exist presently, and it is desirable to assess the capabilities, as well as identify the potential limitations of the methods described herein. 
Based on the numerical experimentation presented we observe that the suite of spectral elementbased algorithms discussed is capable of delivering accurate predictions for the complex BiGlobal EVP at Reynolds numbers of typical relevance to instability analysis. Nevertheless, the question whether the proposed store-and-invert methodology is a viable alternative to the computationally efficient time-stepping approaches [7] still need be addressed in future studies.

\section{APPENDIX A: DETAILS OF THE GALERKIN FORMULATION}

Defining the velocity basis functions as $\psi$ and the pressure basis functions as $\phi$. the following entries of the matrices $A$ and $B$ (for each element) in the different eigenvalue problems appearing in Equations (15), (22) and (33) are obtained

$$
\begin{gathered}
R_{i j}=\int_{\Omega} \frac{\partial \psi_{i}}{\partial x_{m}} \frac{\partial \psi_{j}}{\partial x_{m}} \mathrm{~d} \Omega, \quad i, j=1, \ldots, P+1 \quad m=1,2 . \\
M_{i j}=\int_{\Omega} \psi_{i} \psi_{j} \mathrm{~d} \Omega, \quad i, j=1, \ldots, P+1 . \\
E_{i j}=\int_{\Omega} \bar{u}_{1} \psi_{i} \psi_{j} \mathrm{~d} \Omega, \quad i, j=1, \ldots, P+1 . \\
C_{i j}^{k m}=\int_{\Omega}\left(\frac{\partial \bar{u}_{k}}{\partial x_{m}}\right) \psi_{i} \psi_{j} \mathrm{~d} \Omega, \quad i, j=1, \ldots, P+1 \quad k=1,2,3 \quad m=1,2 . \\
D_{i j}=\int_{\Omega} \phi_{i} \psi_{j} \mathrm{~d} \Omega, \quad i=1, \ldots, P \quad j=1, \ldots, P+1 \\
\lambda_{i j}^{y}=\int_{\Omega} \phi_{i} \frac{\partial \psi_{j}}{\partial y} \mathrm{~d} \Omega, \quad i=1, \ldots, P \quad j=1, \ldots, P+1 . \\
F_{i j}=\int_{\Omega} f(x, y) \psi_{i} \psi_{j} \mathrm{~d} \Omega, \quad i, j=1, \ldots, P+1 .
\end{gathered}
$$

\section{ACKNOWLEDGEMENTS}

This work has been supported by a grant of the Universidad Politécnica de Madrid which make possible a short stay of Dr.González at the Imperial College London. The authors also wish to acknowledge the Colegio-Asociación de Ingenieros del ICAI for the funding and support of this research. Thanks are due to Mr. D. Rodríguez for his contribution in the mathematical description of the curvilinear intakes.

\section{REFERENCES}

1. Peyret R. Spectral Methods for Incompressible Viscons Flow. Springer: Berlin, 2002.

2. Canuto C. Hussaini M, Quarteroni A. Zang T. Spectral Methods in Fluid Dvnamics. Springer: Berlin. 1987.

3. Theofilis V. Advances in global linear instability analysis of nonparallel and three-dimensional fows. Progress in Aerospace Sciences 2003: 39:249-315.

4. Barkley D, Blackbum HM, Sherwin SJ. Direct optimal growth analysis for timesteppers. Intemational Journat for Numerical Methods in Fluids 2008; 57:1435-1458.

5. Karniadakis GE. Sherwin SJ. Spectral/hp Element Methods for Computational Flaid Dynamics (2nd edn). Oxford University Press: Oxford, 2005.

6. Barkley D, Henderson RD. Three-dimensional floquet stability analysis of the wake of a circular cylinder. Joumal of Fluid Mechanics 1996; 322:215-241.

7. Tuckerman L, Barkley D. Bifurcation Analysis for Timesteppers. IMA Volumes in Mathematics and its Applications. Springer: New York, 1999; 453-466.

8. Edwards WS. Tuckerman LS, Friesner RA. Sorensen DC. Krylov methods for the incompressible Navier-Stokes equations. Joumal of Computational Plysics 1994; 110(1):82-102.

9. Theofilis V. Barkley D, Sherwin SJ. Spectral/hp element technology for tow instability and control. Aeronautical Journal 2002; 106:619-625. 
10. Sherwin SJ, Blackburn HM. Three-dimensional instabilities of steady and pulsatile axisymmetric stenotic flows. Journal of Fluid Mechanics 2005; 533:297-327.

11. Abdessemed N. Sherwin SJ. Theofilis V. Linear instability analysis of low pressure turbine fows. Joumal of Fluid Mechanics 2009; 628:57-83.

12. Fietier N, Deville M. Time-dependent algorithms for the simulation of viscoelastic flows with spectral element methods: applications and stability. Journal of Computational Physics 2003; 186:93-121.

13. Kitsios V, Rodriguez D, Theofilis V, Ooi Soria J. Biglobal instability analysis of turbulent tow over an airfoil at an angle of attack. Thirty-eighth Fluid Dynamics Conference and Exhibit. Seattle, Washington. 2008; 9. AIAA, no. Paper 2008-2541.

14. Trefethen L. Spectral Methods in Matlab. SIAM: Philadelphia, 2000.

15. Cuvelier C, Segal A, van Steenhoven AA. Finite Element Methods and Navier-Stokes Equations. D. Reidel Publishing Company: Dordrecht. 1986.

16. González L, Theofilis V. Gómez-Blanco R. Finite-element numerical methods for viscous incompressible biglobal linear instability analysis on unstructured meshes. AlAA Journal 2007; 45(4):840-855.

17. Maday CBY. Approximations Spectrales de Problemes Aux Limites Elliptiques. Springer: Paris, 1992.

18. Anderson E. Bai Z. Bischof C, Blackford S, Demmel J. Dongarra J, Du Croz J, Greenbaum A. Hammarling S. McKenney A, Sorensen D. LAPACK U/sers' Guide (3rd edn). Society for Industrial and Applied Mathematics: Philadelphia. PA, 1999.

19. Kirchner N. Computational aspects of the spectral Galerkin fem for the Orr-Sommerfeld equation. Internationat Joumal for Numerical Methods in Fluids 2000; 32:119-137.

20. Schwab C. $p$ and hp-Finite Element Methods. Theory and Applications in Solid and Fluid Mechanics. Oxford Science Publications: Oxford, U.K., 2004.

21. Theofilis V, Duck PW, Owen J. Viscous linear stability analysis of rectangular duct and cavity flows. Journat of Fluid Mechanics $2004 ; 505: 249-286$.

22. Orszag S. Accurate solution of the orr-sommerfeld stability equation. Journal of Fluid Mechanics 1971: 50: 689-703.

23. Tatsumi T. Yoshimura T. Stability of the laminar flow in a rectangular duct. Journal of Fluid Mechanics 1990; 212:437-449.

24. Leriche E. Gavrilakis S, Deville MO. Direct simulation of the lid-driven cavity fow with chebyshev polynonials. In Proceedings of the 4th European Computationat Fluid Dvnamics Conference (ECCOMAS), Papailiou KD (ed.), Athens, Greece, vol. 1(1). 1998: 220-225.

25. González L, Rodriguez D, Theofilis V. On instability analysis of realistic intake fows. Thirty-serenth AIAA Fluid Dinamics Conference. Seattle, WA. 23-26 Jun 2008; 9. AlAA no. Paper 2004-4380.

26. Schmid P, Henningson DS. Stability and Transition in Shear Flows. Springer: New York, 2001.

27. Salwen H, Cotton FW. Grosch CE. Linear stability of poiseuille fow in a circular pipe. Journal of Fluid Mechanics $1980 ; \mathbf{9 8 : 2 7 3 - 2 8 4 . ~}$

28. Jiménez J. A numerical study of the temporal eigenvalue spectrum of the Blasius boundary layer. Joumal of Fluid Mechanics 1976; 73:497-520.

29. Lessen M. Sadler SG. Liu TY. Stability of pipe poiseuille flow. Phvsics of Fluids 1968; 11:1404-1409.

30. Rosenhead L. Laminar Boundany Lavers. Oxford University Press: Oxford, 1963.

31. Bourcier M, Francois C. Integration numerique des equations de Navier-Stokes dans un domine carré. La Recherche Aerospatiale 1969; 131:23-33.

32. Poliashenko M, Aidun CK. A direct method for computation of simple bifurcations. Joumal of Computational Physics $1995 ; 121: 246-260$.

33. Theofilis V. Globally unstable basic tows in open cavities. AlAA 2000; 2000-1965:12. 